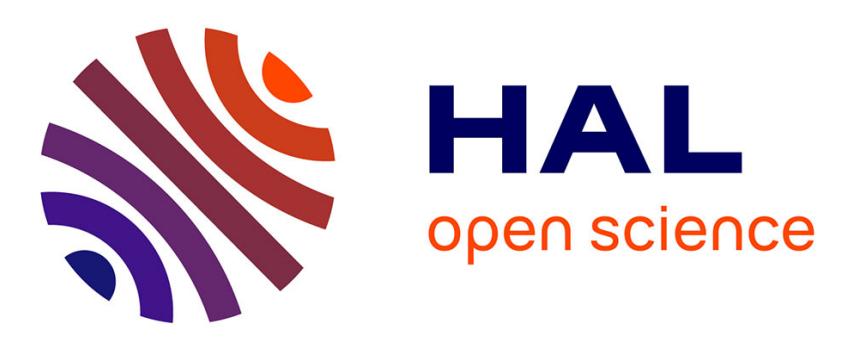

\title{
Action Principles for Quantum Automata and Lorentz Invariance of Discrete Time Quantum Walks
}

\author{
Fabrice Debbasch
}

\section{To cite this version:}

Fabrice Debbasch. Action Principles for Quantum Automata and Lorentz Invariance of Discrete Time Quantum Walks. Annals Phys., 2019, 405, pp.340-364. 10.1016/j.aop.2019.03.005 . hal-01818133

\section{HAL Id: hal-01818133 \\ https://hal.science/hal-01818133}

Submitted on 22 Oct 2021

HAL is a multi-disciplinary open access archive for the deposit and dissemination of scientific research documents, whether they are published or not. The documents may come from teaching and research institutions in France or abroad, or from public or private research centers.
L'archive ouverte pluridisciplinaire HAL, est destinée au dépôt et à la diffusion de documents scientifiques de niveau recherche, publiés ou non, émanant des établissements d'enseignement et de recherche français ou étrangers, des laboratoires publics ou privés. 


\title{
Action Principles for Quantum Automata and Lorentz Invariance of Discrete Time Quantum Walks
}

\author{
Fabrice Debbasch ${ }^{\mathrm{a}, \mathrm{b}}$ \\ ${ }^{a}$ Sorbonne Université, Observatoire de Paris, Université PSL, CNRS, LERMA, F-75005 \\ Paris, France \\ ${ }^{b}$ fabrice.debbasch@sorbonne-universite.fr
}

\begin{abstract}
A discrete action principle for general quantum automata is proposed. This action principle is particularized to Discrete Time Quantum Walks (DTQWs) and then extended into an energy and momentum preserving, manifestly covariant formulation. Space-time coordinates are introduced as new variables of the action and their equations of motion enforce energy and momentum conservation. This guarantees that the proposed action can be used to build future, DTQW-based self-consistent models of spinors interacting with gauge fields. A discrete stress-energy tensor for the DTQW is also obtained. The manifest covariance of the formulation highlights the special role played by the grid reference frame in the DTQW dynamics. The main discussion is complemented by three appendices.
\end{abstract}

Keywords: Discrete time quantum walks, action principles, Lorentz invariance, Dirac equation, quantum information

\section{Introduction}

Discrete Time Quantum Walks (DTQWs) are special unitary quantum automata that can be viewed as formal generalizations of classical random walks. Following the seminal work of Feynman [1] and Aharonov [2] they were considered in a systematic way by Meyer [3]. DTQWs have been realized experimentally with a wide range of physical objects and setups $[4,5,6,7,8$, $9,10]$, and are studied in a large variety of contexts, ranging from quantum optics [10] to quantum algorithmics $[11,12]$, condensed matter physics [13, $14,15,16,17]$ and biophysics [18, 19]. 
It has been shown recently $[20,21,22,23,24,25,26,27,28]$ that several DTQWs can be considered as discrete models of Dirac fermions coupled to arbitrary Yang-Mills gauge fields (including electromagnetic fields) and to relativistic gravitational fields. And a DTQW coupled to a uniform electric field has already been realized experimentally [29]. It is thus tempting to think one could use DTQWs to build new self-consistent discrete models of Dirac fermions interacting with gauge fields, where DTQWs are not only acted upon by gauge fields, but also as sources to these fields. Ideally, such models would have to conserve energy and momentum of the whole system constituted of the DTQWs and the gauge field, and they also would have to be exactly both gauge invariant and covariant. If several DTQWs displaying exact discrete Yang-Mills gauge invariance have already been proposed, the only self-consistent interaction model [26] which has been suggested for an electromagnetic field coupled to a DTQW does not conserve energy [30] and Lorentz invariance of DTQWs is still a debated issue [31, 32, 33].

The aim of this article is to kill these two birds with one stone in constructing manifestly covariant, energy and momentum preserving action principles for DTQWs which can be readily extended to include covariant kinetic terms for the gauge fields.

We first prove that general unitary quantum automata admit a simple energy preserving action principle. We then restrict the discussion to DTQWs with constant coefficients on $(1+1) \mathrm{D}$ space-time grid and use this general action principle to construct densities for the energy and momentum and for their fluxes in the proper frame of the 2D space-time grid.

This action principle, as it stands, presents two limitations. First, energy conservation derives from a particular algebraic property of unitary quantum automata which more general principles involving gauge fields will generically not exhibit, if only because gauge fields are not necessarily charged. Second, Lorentz invariance cannot be properly discussed on this action principle because it does not involve space-time coordinates, but only the discrete labels of grid points.

We therefore construct step by step a new action principle with space-time coordinates as extra variables. This action principle can be written in a manifestly covariant manner and be readily generalized to energy and momentum conserving action principles for DTQWs and gauge fields because energy and momentum conservation is now as consequence of the equations of motion for the space-time coordinates. As a consequence, functional differentiation with respect to the gradients of the space-time coordinates (conceived as 
functions of the grid labels) delivers the stress-energy tensor of the DTQW.

The manifest covariance of the formulation highlights that the grid reference frame plays a special role in the DTQW dynamics and this reference frame enters the equations of motion by its time-like velocity field and its space-like orthogonal.

To sum up the main results, the article brings DTQWs closer to traditional continuum physics by showing that the DTQW dynamics can be derived from action principles which conserve energy-momentum (through conservation equations for the DTQW stress-energy tensor) and display the standard Lorentz symmetry. This is particularly interesting because symmetries and conserved quantities in general are not only mathematically important tools to understand the properties of solutions, but also contribute to the stability of numerical simulations. And knowing the expression of the DTQW conserved stress-energy tensor is a necessary step in coupled the walks to a general relativistic gravitational field

These results are carefully discussed in the last section, and complemented with three Appendices. The first one is a tutorial on discrete variational principles. The second one shows how charge conservation can be recovered from the first action principle The third one revisits the Lorentz invariance of the flat space-time $(1+1) \mathrm{D}$ Dirac equation, with special emphasis on geometry and with extensions to curved space-times in mind. All material presented in the Appendices is new.

\section{Basic action principle}

\subsection{General Quantum Automata}

Consider a discrete time quantum automaton defined in a certain Hilbert space $\mathcal{H}$ by the general abstract evolution equation $\Psi_{j+1}=U_{j} \Psi_{j}$, where $\Psi_{j}$ represents the state of the automaton at time $j$ and $U_{j}$ is a possibly timedependent unitary operator. The Hilbertian product in $\mathcal{H}$ will be denoted by brackets.

This article deals mainly with discrete time quantum walks (DTQWs), which form a special subset of quantum automata. For DTQWs defined on a certain discrete spatial set $L$ (typically a lattice or a graph), $\Psi_{j}$ represents the collection $\left\{\psi_{j p}^{\sigma}, \sigma \in \Sigma, p \in L\right\}$ where $\sigma$ labels spin components and $p$ labels points in $L$. The action of the operator $U_{j}$ on $\Psi_{j}$ takes the typical form:

$$
\left(U_{j} \Psi_{j}\right)_{p}^{\sigma}=\sum_{\sigma^{\prime} \in \Sigma, q \in N_{p}}\left(U_{j p}^{q}\right)_{\sigma^{\prime}}^{\sigma} \psi_{j q}^{\sigma^{\prime}}
$$


where the summation extends to all spin components and to points $q$ located in a certain 'neighborhood' $N_{p}$ of point $p$. The Hilbert product is then

$$
<\Phi_{j} \mid \Psi_{k}>=\sum_{\sigma \in \Sigma, p \in L}\left(\phi_{\sigma}^{\dagger}\right)_{j p} \psi_{k p}^{\sigma}
$$

DTQWs are discrete versions of relativistic quantum mechanics. In particular, the quantity $\Psi_{j, p}$ represents the value taken by the wave-function at discrete time $j$ and discrete position $p$ and $\Psi$ is thus a classical field, not a quantum field. It nevertheless represents discrete quantum physics, exactly as the Schrödinger wave-function in conventional quantum mechanics, which is a classical (as opposed to quantum) field, is used to describe continuous non relativistic quantum physics. The so-called second quantization of DTQWs is briefly discussed in the final section of this article.

The quantum automata dynamics $\Psi_{j+1}=U_{j} \Psi_{j}$ is, by definition, first order in (discrete) time. This article is focused on DTQWs, which form a particular subset of quantum automata and which are closely linked, at the continuous limit, with the Dirac equation, which is also first order in time. It is therefore natural to take inspiration from the Dirac action to build an action for DTQWs. As recalled in Appendix C, the flat space-time free Dirac Lagrangian density reads

$$
\begin{aligned}
L_{D}\left[\Psi, \Psi^{\dagger}\right]= & \left(\Psi^{-}\right)^{*}\left(\partial_{t} \Psi^{-}-\left(U_{D} \Psi\right)^{-}\right) \\
& +\left(\Psi^{+}\right)^{*}\left(\partial_{t} \Psi^{+}-\left(U_{D} \Psi\right)^{+}\right) .
\end{aligned}
$$

where $\left(U_{D} \Psi\right)^{-}=\partial_{x} \Psi^{-}-i m \Psi^{+}$and $\left(U_{D} \Psi\right)^{+}=-\partial_{x} \Psi^{+}-i m \Psi^{-}$. When viewed as a functional of the independent variables $\Psi$ and $\Psi^{\dagger}$, this Lagrangian density leads to the so-called Schrödinger form $\partial_{t} \Psi=U_{D} \Psi$ of the Dirac equation. Consider now, for arbitrary quantum automata, the action

$$
S\left[\Psi, \Psi^{\dagger}\right]=\sum_{j}<\Psi_{j+1} \mid \Psi_{j+1}-U_{j} \Psi_{j}>
$$

viewed as a functional of the independent variables $\Psi=\left\{\Psi_{j}, j \in \mathbb{Z}\right\}$ and $\Psi^{\dagger}=\left\{\Psi_{j}^{\dagger}, j \in \mathbb{Z}\right\}$. Treating $\Psi$ and $\Psi^{\dagger}$ as independent is customary in dealing with action principles for the Klein-Gordon, Dirac, and Proca equations [34] and comes ultimately from the fact that complex conjugation is not a holomorphic function [35]. 
Varying $\Psi$ and $\Psi^{\dagger}$ independently leads to $\delta S=\Delta S+\Delta^{\dagger} S$ with

$$
\Delta S=\sum_{j}<\Psi_{j+1} \mid \delta \Psi_{j+1}-U_{j} \delta \Psi_{j}>
$$

and

$$
\Delta^{\dagger} S=<\delta \Psi_{j+1} \mid \Psi_{j+1}-U_{j} \Psi_{j}>
$$

Now,

$$
\begin{aligned}
\Delta S & =\sum_{j}\left[<\Psi_{j+1}\left|\delta \Psi_{j+1}>-<\Psi_{j+1}\right| U_{j} \delta \Psi_{j}>\right] \\
& =\sum_{j}<\Psi_{j}\left|\delta \Psi_{j}>-\sum_{j}<U_{j}^{\dagger} \Psi_{j+1}\right| \delta \Psi_{j}> \\
& =\sum_{j}<\Psi_{j}-U_{j}^{\dagger} \Psi_{j+1} \mid \delta \Psi_{j}> \\
& =\sum_{j}<U_{j}^{\dagger}\left(U_{j} \Psi_{j}-\Psi_{j+1}\right) \mid \delta \Psi_{j}>
\end{aligned}
$$

where the relation $U_{j}^{\dagger} U_{j}=1$ expressing the fact that $U_{j}$ is unitary has been used to obtain the last equation.

Imposing that $S$ is extremal with respect to arbitrary variations of both $\Psi$ and $\Psi^{\dagger}$ therefore leads to the single equation $\Psi_{j+1}=U_{j} \Psi_{j}$. The functional $S$ can thus be used as action for the quantum automaton defined by $\left\{U_{j}, j \in \mathbb{Z}\right\}$. Note that the action (4), as the Dirac action, is clearly invariant under the global phase transformation $\Psi \rightarrow \exp \left(i \phi_{0}\right) \Psi$ with constant $\phi_{0}$ and thus conserves the charge $Q_{j}=<\Psi_{j} \mid \Psi_{j}>$.

\subsection{Conserved Hamiltonian}

Let us introduce the discrete derivative $\nu_{j}=(D \Psi)_{j}=\Psi_{j+1}-\Psi_{j}$ and rewrite the action $S$ as

$$
\tilde{S}\left[\Psi, \Psi^{\dagger}, \nu\right]=\sum_{j}<\Psi_{j+1} \mid \nu_{j}-\left(U_{j}-1\right) \Psi_{j}>,
$$

where $\Psi_{j+1}^{\dagger}$ has not been expressed in terms of $\nu_{j}^{\dagger}$ to ensure that all discrete equations follow as closely as possible their continuous counterpart valid for the Dirac equation. 
The momentum of $\Psi_{j}$ is $\pi_{j}=\Psi_{j+1}^{\dagger}$ and the momentum of $\Psi_{j}^{\dagger}$ vanishes identically. The Legendre transform of $\tilde{S}_{H}$ reads:

$$
\tilde{S}_{H}[\Psi, \pi]=\sum_{j}<\pi_{j}^{\dagger} \mid\left(U_{j}-1\right) \Psi_{j}>
$$

This function delivers the correct equation of motion in the form

$$
(D \Psi)_{j}=\Psi_{j+1}-\Psi_{j}=+\left(\frac{\partial \tilde{S}}{\partial \pi}\right)_{\mid j}=\left(U_{j}-1\right) \Psi_{j} .
$$

and

$$
(D \pi)_{j-1}=\pi_{j}-\pi_{j-1}=-\left(\frac{\partial \tilde{S}}{\partial \Psi}\right)_{\mid j}=-\pi_{j}\left(U_{j}-1\right)
$$

This second equation can be rewritten $-\pi_{j-1}=-\pi_{j} U_{j}$ or $\pi_{j-1}^{\dagger}=U_{j}^{\dagger} \pi_{j}^{\dagger}$ or, using the unitarity of $U_{j}$ :

$$
U_{j} \pi_{j-1}^{\dagger}=\pi_{j}^{\dagger},
$$

which admits $\pi_{j}^{\dagger}=\Psi_{j+1}$ as solution.

Contrary to the general discrete case (see Appendix A), the Hamiltonian $H_{j}\left(\Psi_{j}, \pi_{j}\right)=<\pi_{j}^{\dagger} \mid\left(U_{j}-1\right) \Psi_{j}>$ obtained from the action by applying a by Legendre transformation is then conserved if $U_{j}=U$ does not depend explicitly on $j$. Indeed, one can then write

$$
\begin{aligned}
H_{j+1} & =<\pi_{j+1}^{\dagger} \mid(U-1) \Psi_{j+1}> \\
& =<U \pi_{j}^{\dagger} \mid(U-1) U \Psi_{j}> \\
& =<\pi_{j}^{\dagger} \mid U^{\dagger}(U-1) U \Psi_{j}> \\
& =<\pi_{j}^{\dagger} \mid(U-1) \Psi_{j}> \\
& =H_{j}
\end{aligned}
$$

where the unitarity of $U$ has been used in passing from the third to the fourth equation.

The Hamiltonian can also be written as $H=<\Psi_{j} \mid\left(1-U^{\dagger}\right) \Psi_{j}>$. Introducing the effective or pseudo-Hamiltonian $h$ of the unitary $U$, one finds $H=<\Psi_{j} \mid(1-\exp (-i h)) \Psi_{j}>$. The conservation of $H$ is thus equivalent to the conservation of the average value of $h$. In the continuous case, $H \simeq$ $i<\Psi_{j}|h| \Psi_{j}>$. Note that $h$ is an operator while $H$ is a scalar function because $H$ is obtained by applying a Legendre transformation to the action, which is itself a scalar. 


\subsection{Energy Conservation for DTQWs on the line}

Let us now focus, for simplicity sakes, on the so-called one-step $(1+1) \mathrm{D}$ DTQWs with two component wave functions $\Psi=\left(\psi^{-}, \psi^{+}\right)^{\dagger}$, for which $U_{j}=$ $V_{j} T$ where $T$ is the $j$-independent spatial translation operator $T$ defined by

$$
\begin{aligned}
& (T \psi)_{p}^{-}=\psi_{p+1}^{-} \\
& (T \psi)_{p}^{+}=\psi_{p-1}^{+}
\end{aligned}
$$

and $V_{j}$ is defined

$$
\left(V_{j} \Psi\right)_{p}=W_{j, p} \Psi_{p}
$$

with $W_{j, p}$ an arbitrary $j$ - and $p$-dependent operator in $U(2)$.

Consider the following alternate form of the action $\tilde{S}$ :

$$
\tilde{S}\left[\Psi, \Psi^{\dagger}, \nu\right]=\sum_{j}<\Psi_{j} \mid U^{\dagger} \nu_{j}-\left(1-U^{\dagger}\right) \Psi_{j}>,
$$

which can be obtained from (8) by taking into account that $\Psi_{j+1}=U \Psi_{j}$. This alternate form is completely equivalent to the original form but it is in practice more useful because it delivers the Hamiltonian directly under the form $H_{j}=<\Psi_{j} \mid\left(1-U_{j}^{\dagger}\right) \Psi_{j}>$, which is easier to use than the expression derived from (8). However, (4) and (8) are easier to guess from the Dirac action than (16). This is why this presentation starts with (4) and (8), and not with (16).

A straightforward computation shows that the energy density corresponding to the Hamiltonian $H_{j}=<\Psi_{j} \mid\left(1-U_{j}^{\dagger}\right) \Psi_{j}>$ reads

$$
\mathcal{H}_{j, p}=\Psi_{j, p}^{\dagger}\left(\left(1-U^{\dagger}\right) \Psi_{j}\right)_{p}
$$

and obeys the conservation equation

$$
\left(\nabla_{j} \mathcal{H}\right)_{j, p}+\left(\nabla_{p} \mathcal{J}_{\mathcal{H}}\right)_{j, p}=0
$$

with

$$
\left(\mathcal{J}_{\mathcal{H}}\right)_{j, p}=-\Psi_{j, p}^{\dagger} \sigma_{3}\left(\left(1-U^{\dagger}\right) \Psi_{j}\right)_{p} .
$$

Here, $\sigma_{3}$ is the third Pauli matrix i.e. $\sigma_{3}=\operatorname{diag}(1,-1)$ and

$$
\left(\nabla_{j} \mathcal{H}\right)_{j, p}=\mathcal{H}_{j+1, p}-\frac{\mathcal{H}_{j, p+1}+\mathcal{H}_{j, p-1}}{2},
$$




$$
\left(\nabla_{p} \mathcal{J}_{\mathcal{H}}\right)_{j, p}=\frac{\left(\mathcal{J}_{\mathcal{H}}\right)_{j, p+1}-\left(\mathcal{J}_{\mathcal{H}}\right)_{j, p-1}}{2} .
$$

Note that $\mathcal{H}_{j, p}=Q_{j, p}+h_{j, p}$, where $Q_{j, p}=\Psi_{j, p}^{\dagger} \Psi_{j, p}$ is the local charge density and $h_{j, p}=-\Psi_{j, p}^{\dagger}\left(U^{\dagger} \Psi_{j}\right)_{p}$. The current $\left(\mathcal{J}_{\mathcal{H}}\right)_{j, p}$ accordingly splits into two contributions, the charge current $\left(\mathcal{J}_{Q}\right)_{j, p}=-\Psi_{j, p}^{\dagger} \sigma_{3} \Psi_{j, p}([26])$ and the current $\left(\mathcal{J}_{h}\right)_{j, p}=\Psi_{j, p}^{\dagger} \sigma_{3}\left(U^{\dagger} \Psi_{j}\right)_{p}$.

We now define the momentum $\mathcal{P}$ of the DTQW by its spatial 'density'

$$
\mathcal{P}_{j, p}=\frac{1}{2} \Psi_{j, p}^{\dagger} \sigma_{3}\left(\left(T-T^{\dagger}\right) \Psi_{j}\right)_{p} .
$$

This quantity is conserved if all coefficients of the DTQW are constants, as can be easily seen by writing the DTQW evolution equations in Fourier space, and also coincides, in the continuous limit, with the wave number of the walk. The associated momentum current is

$$
\left(\mathcal{J}_{\mathcal{P}}\right)_{j, p}=-\frac{1}{2} \Psi_{j, p}^{\dagger}\left(\left(T-T^{\dagger}\right) \Psi_{j}\right)_{p} .
$$

In working with the alternate form of the action, it is useful to change variables i.e. to replace the independent variables $\Psi$ and $\Psi^{\dagger}$ by the variables $\Phi=U^{\dagger} \Psi$ and $\Psi^{\dagger}=(U \Phi)^{\dagger}$. The action then reads:

$$
\tilde{S}\left[\Phi, \Psi^{\dagger}, \nu^{\Phi}\right]=\sum_{j}<\Psi_{j} \mid \nu_{j}^{\Phi}-(U-1) \Phi_{j}>,
$$

where $\nu_{j}^{\Phi}=\Phi_{j+1}-\Phi_{j}$ is the new velocity.

The conserved Hamiltonian and momentum as well as their spatial densities and currents can also be expressed in terms of these variables. The Hamitonian $H_{j}$ reads

$$
H_{j}=<\Psi_{j} \mid(U-1) \Phi_{j}>,
$$

its density $\mathcal{H}_{j}$ is therefore

$$
\mathcal{H}_{j, p}=\Psi_{j, p}^{\dagger}((U-1) \Phi)_{j, p}
$$

and its current $\mathcal{J}_{j, p}$ is

$$
\mathcal{J}_{j, p}=-\Psi_{j, p}^{\dagger} \sigma_{3}((U-1) \Phi)_{j, p} .
$$


The momentum $P_{j}$ reads

$$
P_{j}=\frac{1}{2}<\Psi_{j} \mid \sigma_{3}\left(T-T^{\dagger}\right) U \Phi_{j}>
$$

its density $\mathcal{P}_{j, p}$ is therefore

$$
\mathcal{P}_{j, p}=\frac{1}{2} \Psi_{j, p}^{\dagger} \sigma_{3}\left(\left(T-T^{\dagger}\right) U \Phi\right)_{j, p}
$$

and its current $\mathcal{J}_{\mathcal{P}_{j, p}}$ is

$$
\left(\mathcal{J}_{\mathcal{P}}\right)_{j, p}=-\frac{1}{2} \Psi_{j, p}^{\dagger}\left(\left(T-T^{\dagger}\right) U \Phi\right)_{j, p} .
$$

\section{Extended Action Principle for DTQWs on the line}

Not all DTQWs admit a continuous limit and the physical interpretation of the limit depends on the unitary $U$. When $U$ is constant, the limit, if it exists, describes a free Dirac particle. When $U$ is not constant, the limit essentially describes a Dirac particle acted on by gauge fields. For DTQWs with constant unitaries, the dynamics derived from the action principle introduced in the previous section conserves the Hamiltonian $H$ and the momentum $P$ of DTQWs. Though this result is true even for DTQWs which do not admit a continuous limit, its interpretation for the walks which do admit a limit is clear: these walks describe a free particle whose energy and momentum is evidently conserved.

Consider now DTQWs with non constant unitaries. The continuous limit, when it exists, describes Dirac particles acted on by gauge fields and, even outside this limit, several classes of DTQWs exhibit exact discrete gauge invariances, and the interpretation of these DTQWs as Dirac particles acted on by discrete gauge fields thus makes perfect sense. The Hamiltonian and momentum of the DTQWs with non constant unitaries is not conserved. The obvious interpretation is that the particle now exchanges energy and momentum with the gauge fields and, in continuous physics, the conserved quantities are indeed the sum of the energy and momentum of the DTQWs with the energy and momentum of the fields.

Suppose one wants to define an energy and momentum for the discrete gauge fields acting on the DTQWs. The natural way to proceed is to extend 
the above DTQW action by adding a kinetic term for the discrete gauge fields, using for example the discrete field strengths $[26,36]$ and to derive from this discrete action a discrete Hamiltonian. As explained in Appendix A, this discrete Hamiltonian will not be generically conserved by the discrete dynamics. This is so because the dynamics of the gauge fields is not as simple as the DTQW dynamics and follows the general rule of non conserving the discrete Hamiltonian. Thus, if one extends the above DTQW action as it is to include kinetic terms for discrete gauge fields, the program of building discrete actions which couple self-consistently DTQWs to discrete gauge fields in an energy- and momentum-conserving manner fails. So, in a sense, no discrete gauge theories based on DTQWs if one follows this route.

As explained in Appendix A, the solution is to introduce in the action which describes the combined dynamics of the DTQWs coupled to gauge fields new dynamical variables which represent continuous space-time coordinates. As a preparatory step, this article shows how one can introduce continuous space-time coordinates in the pure DTQW action and recover from this extended DTQW action the energy and momentum conservation laws obtained in the previous section. Using the extended DTQW action, these conservation laws appear as the equations of motion obeyed by the space-time coordinates (which have been introduced as new dynamical variables of the action and thus, by definition, have their own dynamics).

But the extended DTQW action principle presented in this section is not

preparatory work only. Indeed, once coordinates have been introduced as functions defined on the grid, it becomes possible to modify these functions and, in particular, it becomes possible to perform Lorentz transformations on these coordinates without changing the discrete grid. That Lorentz transformations must indeed be carried out on the newly introduced space-time coordinates and not on the grid labels $j$ and $p$ makes sense because, as mentioned in Appendix A, the physical coordinates are represented, not by the grid labels, but by the newly introduced coordinates. This will be explored in the next section.

\subsection{Intrinsic geometry on the grid}

We first make explicit the fact that the operator $T$ codes for spatial discrete derivatives by writing

$$
T=\sigma_{3} \nabla_{p}+C
$$


where

$$
\left(\nabla_{p} \Phi\right)_{j, p}=\frac{1}{2}\left(\Phi_{j, p+1}-\Phi_{j, p-1}\right)
$$

and

$$
(C \Phi)_{j, p}=\frac{1}{2}\left(\Phi_{j, p+1}+\Phi_{j, p-1}\right) .
$$

In Fourier space for a wave vector $k$, the operator $\nabla_{p}$ delivers a $i \sin k$ factor, which becomes $i k$ i.e. a derivation in the continuous limit, and the operator $C$ delivers a $\cos k$ factor, which becomes unity in the same limit.

We also introduce the discrete time derivative operator $\nabla_{j}$ defined by

$$
\left(\nabla_{j} \Phi\right)_{j, p}=\left(\Phi_{j+1, p}-\Phi_{j, p}\right)=\left(\nu_{j}^{\Phi}\right)_{j, p}
$$

and use the definition $U=W T$ to write the action $\tilde{S}$ from (24) under the form

$$
\begin{aligned}
\tilde{S}\left[\Phi, \Psi^{\dagger}, \nabla_{j} \Phi, \nabla_{p} \Phi\right] & =\sum_{j}<\Psi_{j} \mid\left(\nabla_{j} \Phi\right)_{j}-W \sigma_{3}\left(\nabla_{p} \Phi\right)_{j} \\
& -(W C-1) \Phi_{j}>_{p},
\end{aligned}
$$

where the notation $<A \mid B>_{p}=\sum_{p} A_{p}^{*} B_{p}$ has been used.

The conserved Hamiltonian $H_{j}(25)$ reads

$$
\begin{aligned}
H_{j} & =<\Psi_{j} \mid(U-1) \Phi_{j}>_{p} \\
& =<\Psi_{j} \mid\left(W \sigma_{3} \nabla_{p}+W C-1\right) \Phi_{j}>_{p}
\end{aligned}
$$

and the conserved momentum $P_{j}(28)$ reads

$$
\begin{aligned}
P_{j} & =\frac{1}{2}<\Psi_{j} \mid \sigma_{3}\left(T-T^{\dagger}\right) U \Phi_{j}>_{p} \\
& =<\Psi_{j} \mid \nabla_{p} U \Phi_{j}>_{p} \\
& =<\Psi_{j} \mid U \nabla_{p} \Phi_{j}>_{p}
\end{aligned}
$$

\subsection{Space-time coordinates}

The extended action $S_{e}$ involves two additional variables. These are a continuous time variable $t_{j, p}=X_{j, p}^{0}$ and a continuous space variable $x_{j, p}=X_{j, p}^{1}$. If one follows the procedure outlined in Appendix A, a key step in obtaining the action $S_{e}$ from $S$ is to replace $\nabla_{j}$ and $\nabla_{p}$ by discrete versions $\nabla_{t}=\nabla_{0}$ and $\nabla_{x}=\nabla_{1}$ of the time- and space-derivatives. These are constructed as 
follows. First suppose that $j$ and $p$ were actually continuous coordinates. One could then write

$$
\begin{aligned}
\frac{\partial}{\partial t} & =\frac{\partial j}{\partial t} \frac{\partial}{\partial j}+\frac{\partial p}{\partial t} \frac{\partial}{\partial p} \\
\frac{\partial}{\partial x} & =\frac{\partial j}{\partial x} \frac{\partial}{\partial j}+\frac{\partial p}{\partial x} \frac{\partial}{\partial p}
\end{aligned}
$$

The coefficients in front of $\frac{\partial}{\partial j}$ and $\frac{\partial}{\partial p}$ are those of the Jacobian $\partial(j, p) / \partial(t, x)$. This Jacobian is the inverse of $\partial(t, x) / \partial(j, p)$, form which one deduces

$$
\begin{aligned}
& \frac{\partial j}{\partial t}=+\frac{1}{\delta} \frac{\partial x}{\partial p} \\
& \frac{\partial j}{\partial x}=-\frac{1}{\delta} \frac{\partial t}{\partial p} \\
& \frac{\partial p}{\partial t}=-\frac{1}{\delta} \frac{\partial x}{\partial j} \\
& \frac{\partial p}{\partial x}=+\frac{1}{\delta} \frac{\partial t}{\partial j}
\end{aligned}
$$

where

$$
\delta=\frac{\partial t}{\partial j} \frac{\partial x}{\partial p}-\frac{\partial t}{\partial p} \frac{\partial x}{\partial j}
$$

This suggests that the building blocks from which an extended, coordinate dependent DTQW action can be constructed are

$$
\begin{aligned}
C_{0}^{j} & =+\frac{1}{\Delta(\nabla X)} \nabla_{p} X^{1} \\
C_{0}^{p} & =-\frac{1}{\Delta(\nabla X)} \nabla_{j} X^{1} \\
C_{1}^{j} & =-\frac{1}{\Delta(\nabla X)} \nabla_{p} X^{0} \\
C_{1}^{p} & =+\frac{1}{\Delta(\nabla X)} \nabla_{j} X^{0}
\end{aligned}
$$

with

$$
\Delta(\nabla X)=\left(\nabla_{j} X^{0}\right)\left(\nabla_{p} X^{1}\right)-\left(\nabla_{p} X^{0}\right)\left(\nabla_{j} X^{1}\right)
$$


where

$$
\begin{aligned}
\left(\nabla_{j} X^{\mu}\right)_{j, p} & =\frac{X_{j, p-1}^{\mu}+X_{j, p+1}^{\mu}}{2}-X_{j-1, p}^{\mu} \\
\left(\nabla_{p} X^{\mu}\right)_{j, p} & =\frac{X_{j, p+1}^{\mu}-X_{j, p-1}^{\mu}}{2}
\end{aligned}
$$

One could hope that replacing $\nabla_{j}\left(\right.$ resp. $\left.\nabla_{p}\right)$ by $\nabla_{0}=C_{0}^{j} \nabla_{j}+C_{0}^{p} \nabla_{p}$ (resp. $\nabla_{1}=C_{1}^{j} \nabla_{j}+C_{1}^{p} \nabla_{p}$ ) in the original action (35) delivers a correct, coordinate-dependent extended action, but this simple procedure turns out to be a bit too naive. The right extended action is presented in the next section.

\subsection{Action with coordinates and conserved quantities}

Consider the following action:

$$
\Sigma\left[\Phi, \Psi^{\dagger}, \nabla \Phi, \nabla X\right]=\sum_{j}\left(K_{j}+M_{j}\right)
$$

where the 'mass' term is

$$
M_{j}=M_{j}^{1}+M_{j}^{2}+M_{j}^{3}
$$

with

$$
\begin{gathered}
M_{j}^{1}=<\Psi_{j} \mid(1-W C) \frac{1+T}{2} \Delta\left((\nabla X)_{j}\right) \Phi_{j}>_{p}, \\
M_{j}^{2}=<\Psi_{j} \mid(1-W C) \frac{1-T}{2}\left(\left(\nabla_{j} X^{0}\right)_{j}-\left(\nabla_{p} X^{1}\right)_{j}\right) \Phi_{j}>_{p}, \\
M_{j}^{3}=<\Psi_{j} \mid(1-W C) \frac{1-T}{2} \Phi_{j}>_{p},
\end{gathered}
$$

and the more complicated 'kinetic' term is

$$
K_{j}=K_{j}^{j}+K_{j}^{p}+K_{j}^{\mathrm{supp}}
$$

with

$$
\begin{gathered}
K_{j}^{j}=-<\Psi_{j} \mid W \sigma_{3}\left(\nabla_{j} \chi\right)_{j}\left(\nabla_{p} \Phi\right)_{j}>_{p} \\
K_{j}^{p}=+<\Psi_{j} \mid \sigma_{3}\left(\nabla_{p} \chi\right)_{j}\left(\nabla_{j} \Phi\right)_{j}>_{p} \\
K_{j}^{\text {supp }}=+<\Psi_{j} \mid(1-T)\left(\nabla_{j} \Phi\right)_{j}>_{p}
\end{gathered}
$$


where

$$
\chi=X^{0} \mathbb{1}+X^{1} \sigma_{3} T .
$$

The unitary operators $W$ and $T$ are those entering the definition of the DTQW given above.

Let us now prove that the equations of motion derived from this action are solved by $X_{j, p}^{0}=j, X_{j, p}^{1}=p$ and the original DTQW dynamics $\left(\nabla_{j} \Phi\right)_{j, p}=$ $(U-1) \Phi_{j, p}$. To do this, we first note that the action $\Sigma(44)$ coincides with the action $(35)$ for $(\Phi, \Psi)$ when $X_{j, p}^{0}=j$ and $X_{j, p}^{1}=p$. Thus, the equations of motion for $\Phi$ and $\Psi$ derived from $\Sigma$ are solved by $X_{j, p}^{0}=j, X_{j, p}^{1}=p$, $\left(\nabla_{j} \Phi\right)_{j, p}=(U-1) \Phi_{j, p}$. Note that $M_{j}^{2}$ vanishes identically when $X_{j, p}^{0}=j$ and $X_{j, p}^{1}=p$.

Let us now investigate the equations of motion for the coordinates $X^{0}$ and $X^{1}$ derived from $\Sigma$. The functional derivatives of $\Sigma$ with respect to the gradients of the coordinates read:

$$
\begin{aligned}
\frac{\delta \Sigma}{\delta\left(\left(\nabla_{j} X^{0}\right)_{j, p}\right)} & =\Psi_{j, p}^{\dagger}\left(-W \sigma_{3}\left(\nabla_{p} \Phi\right)_{j, p}\right. \\
& +(1-W C) \frac{1-T}{2} \Phi_{j, p} \\
& \left.+(1-W C) \frac{1+T}{2}\left(\nabla_{p} X^{1}\right)_{j, p} \Phi_{j, p}\right), \\
\frac{\delta \Sigma}{\delta\left(\left(\nabla_{p} X^{0}\right)_{j, p}\right)} & =\Psi_{j, p}^{\dagger}\left(\sigma_{3}\left(\nabla_{j} \Phi\right)_{j, p}\right. \\
& \left.-(1-W C) \frac{1+T}{2}\left(\nabla_{j} X^{1}\right)_{j, p} \Phi_{j, p}\right), \\
\frac{\delta \Sigma}{\delta\left(\left(\nabla_{j} X^{1}\right)_{j, p}\right)} & =\Psi_{j, p}^{\dagger}\left(-U\left(\nabla_{p} \Phi\right)_{j, p}\right. \\
& \left.-(1-W C) \frac{1+T}{2}\left(\nabla_{p} X^{0}\right)_{j, p} \Phi_{j, p}\right),
\end{aligned}
$$

and

$$
\begin{aligned}
\frac{\delta \Sigma}{\delta\left(\left(\nabla_{p} X^{1}\right)_{j, p}\right)} & =\Psi_{j, p}^{\dagger}\left(T\left(\nabla_{j} \Phi\right)_{j, p}\right. \\
& -(1-W C) \frac{1-T}{2} \Phi_{j, p} \\
& \left.+(1-W C) \frac{1+T}{2}\left(\nabla_{j} X^{0}\right)_{j, p} \Phi_{j, p}\right)
\end{aligned}
$$


Evaluating these quantities for $X_{j, p}^{0}=j, X_{j, p}^{1}=p$ and $\left(\nabla_{j} \Phi\right)_{j, p}=(U-$ 1) $\Phi_{j, p}$ leads to

$$
\begin{gathered}
\frac{\delta \Sigma}{\delta\left(\left(\nabla_{j} X^{0}\right)_{j, p}\right)} \hat{=}-\mathcal{H}_{j, p}, \\
\frac{\delta \Sigma}{\delta\left(\left(\nabla_{p} X^{0}\right)_{j, p}\right)} \widehat{=}-\left(\mathcal{J}_{\mathcal{H}}\right)_{j, p}, \\
\frac{\delta \Sigma}{\delta\left(\left(\nabla_{j} X^{1}\right)_{j, p}\right)} \hat{=}-\mathcal{P}_{j, p} \\
\frac{\delta \Sigma}{\delta\left(\left(\nabla_{p} X^{1}\right)_{j, p}\right)} \hat{=}-\left(\mathcal{J}_{\mathcal{P}}\right)_{j, p}
\end{gathered}
$$

where $\widehat{=}$ has been used to indicate are valid only for this particular choice of variables. These equalities show that the equations of motion for the coordinates $X^{0}$ and $X^{1}$ derived from $\Sigma$, when evaluated for $X_{j, p}^{0}=j, X_{j, p}^{1}=p$ and $\left(\nabla_{j} \Phi\right)_{j, p}=(U-1) \Phi_{j, p}$, are identical to the energy and momentum conservation equations obtained from the original DTQW dynamics $\left(\nabla_{j} \Phi\right)_{j, p}=$ $(U-1) \Phi_{j, p}$. This shows that $X_{j, p}^{0}=j, X_{j, p}^{1}=p$ and $\left(\nabla_{j} \Phi\right)_{j, p}=(U-1) \Phi_{j, p}$ solves all the equations of motion derived from the extended action $\Sigma$. As is customary in working with action principles, we will designate this choice of variables as the 'shell'. Thus, for example, to obtain the equations of motion from the action, one has to consider the action 'off-shell'. And the equations of motion are the equations defining the shell.

At this stage, it may seem that the action $\Sigma$ brings little to the table, except unnecessary complications. As indicated earlier, this point of view is mistaken. Indeed, $\Sigma$ contains the space-time coordinates $X^{0}$ and $X^{1}$ as variables defined on the grid and this will allow us to change these variables (without of course modifying the grid) and thus, ultimately, to perform Lorentz transformations. This will be investigated in the next sections. The second advantage of $\Sigma$, namely the fact that energy-and momentum conservation are now recovered as the equations of motion of the space-time coordinates, can be used to build energy-and momentum-conserving actions for gauge fields coupled to DTQWs and will be investigated in forthcoming publications.

\subsection{Lorentz invariance I: main kinetic term}

We first stress that the appearance of $X^{0}$ and $X^{1}$ in the action $\Sigma$ does not mean that $\Sigma$ is valid in an arbitrary Lorentz frame. Indeed, because the 
equations of motion derived from $\Sigma$ are solved by $X^{0}=j$ and $X^{1}=p, \Sigma$ is actually valid in the rest frame of the grid only. Note that the conserved quantities derived from $\Sigma$ are also the energy and momentum in the rest frame of the grid. Our main task is thus to define Lorentz transformations for DTQWs in such a way that (i) these Lorentz transformations coincide with usual continuous Lorentz transformations in the continuous limit (ii) it is possible to find a covariant action which coincides with $\Sigma$ in the rest frame of the grid.

The first step consists in identifying in $\Sigma$ a main kinetic term which ressembles the kinetic from the flat space-time Dirac equation. We will use this term to define Lorentz transformations on DTQWs and then introduce a covariant form for this main kinetic term.

The second step, which will be presented in the next section, consists in dealing with the mass terms and with the extra kinetic terms which vanish in the continuous limit and thus do not contribute to the main kinetic term studied in this section.

The operator $W \sigma_{3}$ is in $U(2)$. It therefore admits two eigenvalues of unit modulus, which we call $\exp \left(i \alpha_{L}\right)$ and $\exp \left(i \alpha_{R}\right)$. We now switch to a new spin basis $\left(b_{f}\right)=\left(b_{L}, b_{R}\right)$ made of normalized eigenvectors of $W \sigma_{3}$. In this new basis, the operator $\sigma_{3}$ is not represented by the third Pauli matrix. We therefore introduce the new operator $\bar{\sigma}_{3}$ whose representation in $\left(b_{f}\right)$ coincides with the third Pauli matrix and write $\sigma_{3} T=\left(\bar{\sigma}_{3}+\left(\sigma_{3}-\bar{\sigma}_{3}\right)\right)(1+(T-1))$, which leads to $\chi=\bar{\chi}+\Delta \chi$ with

$$
\begin{aligned}
\bar{\chi}= & X^{0}+X^{1} \bar{\sigma}_{3} \\
\Delta \chi= & X^{1}\left[\left(\sigma_{3}-\bar{\sigma}_{3}\right)+\bar{\sigma}_{3}(T-1)\right. \\
& \left.+\left(\sigma_{3}-\bar{\sigma}_{3}\right)(T-1)\right]
\end{aligned}
$$

Similarly, $\xi=\sigma_{3} \chi=X^{0} \sigma_{3}+X^{1} T=\bar{\xi}+\Delta \xi$ with

$$
\begin{aligned}
\bar{\xi} & =X^{0} \bar{\sigma}_{3}+X^{1} \\
\Delta \xi & =X^{0}\left(\sigma_{3}-\bar{\sigma}_{3}\right)+X^{1}(T-1)
\end{aligned}
$$

Inserting the above expressions into the kinetic terms of the action $\Sigma$ delivers expressions of the form $K_{j}^{j / p}=\bar{K}_{j}^{j / p}+\Delta K_{j}^{j / p}$ with

$$
\begin{gathered}
\bar{K}_{j}^{j}=-<\Psi_{j} \mid W \sigma_{3}\left(\nabla_{j} \bar{\chi}\right)_{j}\left(\nabla_{p} \Phi\right)_{j}>_{p} \\
\bar{K}_{j}^{p}=+<\Psi_{j} \mid\left(\nabla_{p} \bar{\xi}\right)_{j}\left(\nabla_{j} \Phi\right)_{j}>_{p} .
\end{gathered}
$$


The sum $\bar{K}_{j}=\bar{K}_{j}^{j}+\bar{K}_{j}^{p}$ can be written as $\bar{K}_{j}=\sum_{p} \bar{K}_{j, p}$ with

$$
\bar{K}_{j, p}=\eta_{f g}\left(\Psi^{f}\right)_{j, p}^{*}\left[\left(e_{a}^{q} \gamma^{0} \gamma^{a} D_{q}\right)_{h}^{g} \Phi^{h}\right]_{j, p} \Delta\left((\nabla X)_{j, p}\right)
$$

with $D_{j}=\nabla_{j}, D_{p}=W \sigma_{3} \bar{\sigma}_{3} \nabla_{p}, e_{a}^{q}=C_{a}^{q}($ see $(41))$,

$$
\begin{aligned}
& \left(\gamma^{0}\right)_{g}^{f}=\left(\begin{array}{ll}
0 & 1 \\
1 & 0
\end{array}\right) \\
& \left(\gamma^{1}\right)_{g}^{f}=\left(\begin{array}{cc}
0 & 1 \\
-1 & 0
\end{array}\right),
\end{aligned}
$$

and summation is implied over repeated indices $\{f, g, h\} \in\{L, R\}^{3}, q \in$ $\{j, p\}^{2}$ and $a \in\{0,1\}$. The coefficients $\left(e_{a}^{q}=C_{a}^{q}\right)$ can be interpreted as the $(j, p)$ 'components' of an $X$-dependent 2-bein $\left(e_{0}, e_{1}\right)$ with $\mu$-components $e_{0}^{0}=1=e_{1}^{1}, e_{0}^{1}=e_{1}^{0}=0$ (see Section 'Space-time coordinates' above as well as Appendix C). Introducing $D_{\mu}=C_{\mu}^{j} D_{j}+C_{\mu}^{p} D_{p}$ helps bring $\bar{K}_{j, p}$ under the form

$$
\bar{K}_{j, p}=\eta_{f g}\left(\Psi^{f}\right)_{j, p}^{*}\left[\left(e_{a}^{\mu} \gamma^{0} \gamma^{a} D_{\mu}\right)_{h}^{g} \Phi^{h}\right]_{j, p} \Delta\left((\nabla X)_{j, p}\right),
$$

which is similar to the usual form of the kinetic term in the Dirac equation. We thus define the DTQW Lorentz transformation exactly as the continuous one and we proceed in the same exact way. In particular, the basis vector $b_{L}$ $\left(\right.$ resp. $b_{R}$ ) gets multiplied by a positive real number $\lambda\left(\right.$ resp. $\left.\lambda^{-1}\right)$ and this change of basis is compensated by a change of coordinates through a change of spin metric and of 2-bein. The volume 'measure' $\Delta(\nabla X) \Delta j \Delta p=\Delta(\nabla X)$ is invariant under Lorentz transformation because the coordinates $X^{0}$ and $X^{1}$ in a Lorentz frame of velocity $V$ with respect to the grid frame are given by the usual linear expressions:

$$
\begin{aligned}
& X_{j, p}^{0}=\gamma(V)(j-V p) \\
& X_{j, p}^{1}=\gamma(V)(p-V j)
\end{aligned}
$$

where $\gamma(V)=\left(1-V^{2}\right)^{-1 / 2}$. Thus, in the $\left(X^{0}, X^{1}\right)$ plane, curves of constant grid-time $j$ are straight lines of slope $-1 / V$ where one grid spacestep $\Delta p=1$ corresponds to $\Delta X^{0}=-\gamma(V) V$ and $\Delta X^{1}=+\gamma(V)$. Similarly, curves of constant grid-position $p$ are straight lines of slope $-V$ where one grid time-step $\Delta j=1$ corresponds to $\Delta X^{0}=+\gamma(V)$ and $\Delta X^{1}=$ $-\gamma(V) V$. For arbitrary $V$, these straight lines are not orthogonal for the 
Euclidean metric $\left(d X^{0}\right)^{2}+\left(d X^{1}\right)^{2}$, but rather for the Minkovski (pseudo)metric $\left(d X^{0}\right)^{2}-\left(d X^{1}\right)^{2}$. Note finally that the Lorentz transformation of $\bar{K}_{j}$ is computationally simple because we are working in the basis which makes diagonal $W \sigma_{3}, \bar{\sigma}_{3}$, and thus also their product $W \sigma_{3} \bar{\sigma}_{3}$. Choosing the basis $\left(b_{f}\right)$ orthonormal ensures that the spin-metric is diagonal too.

\subsection{Lorentz invariance II: extra kinetic terms and mass terms}

As a prolegomenon, let us remark that, as any quantity appearing in a covariant theory, an arbitrary spin operator can be fully defined by its value in a particular spin-basis together with its transformation law. This way of defining spin-operators will be used extensively in what follows where most spin operators will be defined by their values in the spin basis corresponding to the grid reference frame combined with the SOLT law introduced in Appendix $\mathrm{C}$ to discuss Lorentz invariance for the Dirac equation.

There are three extra kinetic terms i.e. $\Delta K^{j}, \Delta K^{p}$ and $K^{\mathrm{supp}}$, and two mass terms i.e. $M^{1}$ and $M^{2}$. The mass terms are somewhat simpler and we start with them.

Since $\Delta\left((\nabla X)_{j, p}\right)$ is Lorentz invariant, the first mass-term $M_{j}^{1}=\sum_{p} M_{j, p}^{1}$ can be written in the covariant form

$$
M_{j, p}^{1}=\eta_{f g}\left(\Psi^{f}\right)_{j, p}^{*}\left(\left(m^{1}\right)_{h}^{g} \Phi^{h}\right)_{j, p} \Delta\left((\nabla X)_{j, p}\right)
$$

where the operator $m^{1}=(1-W C) \frac{1+T}{2}$ in the spin basis of the grid frame and obeys the SOLT law.

The second mass term is conceptually more complicated. To write in a manifestly covariant manner, let us introduce the 'vector field' (resp. 'form field') $U^{\mu}$ (resp. $U_{\mu}$ ) is defined by its components $U^{0}=1, U^{1}=0$ (resp. $\left.U_{0}=1, U_{1}=0\right)$ in the grid frame, together with the usual Lorentz transformation law for vector fields (resp. form-fields). In physical terms, $U$ represents the velocity field of the grid in $2 D$ space-time. It also coincides with the first vector of the 2-bein associated to the grid coordinates (see the definition of the coordinate-dependent 2-beins in the previous section). Note that the grid being independent of the choice of coordinates, $\nabla_{j}$ behaves like a scalar. In usual continuous geometrical terms, $\nabla_{j}=U^{\nu} \nabla_{\nu}$. This scalar depends on $U$ i.e. on the choice of grid, not on the choice of coordinates. We also introduce the 'vector field' $V^{\mu}$ defined by its components $V^{0}=0$ and $V^{1}=1$ in the grid frame, together with its associated form $V_{\mu}$. This vector field coincides with the second vector of the 2-bein associated to the 
grid coordinates and will be used immediately below to express one of the extra kinetic terms in a manifestly covariant manner. And the operator $\nabla_{p}$ is a scalar, discrete equivalent to $V^{\nu} \nabla_{\nu}$. Note that contracting a tensor with $V$ essentially amounts to projecting the tensor on the orthogonal to $U$ :

$$
\begin{aligned}
\Pi_{\mu \nu} A^{\nu} & =\left(\eta_{\mu \nu}-U_{\mu} U_{\nu}\right) A^{\nu} \\
& =\left(\eta_{\mu \nu}-U_{\mu} U_{\nu}\right)\left((A . U) U^{\nu}+(A . V) V^{\nu}\right) \\
& =(A . V) V_{\mu},
\end{aligned}
$$

where $\eta_{\mu \nu}$ is the Minkovski metric in orthonormal coordinates, $\Pi$ is the projector unto the orthornomal to $U$ and $(A . B)=\eta_{\alpha \beta} A^{\alpha} B^{\beta}$.

The 'vector fields' $U$ and $V$ help write $M_{j}^{2}=\sum_{p} M_{j, p}^{2}$ with

$$
M_{j, p}^{2}=\eta_{f g}\left(\Psi^{f}\right)_{j, p}^{*}\left(\left(m^{2}\right)_{h}^{g} \Phi^{h}\right)_{j, p}\left(U_{\mu} \nabla_{j}+V_{\mu} \nabla_{p}\right) X^{\mu}
$$

where the spin-operator $m^{2}$ obeys the SOLT law and coincides with (1 $W C) \frac{1-T}{2}$ in the spin basis of the grid frame.

Similarly, $M_{j}^{3}=\sum_{p} M_{j, p}^{3}$ with

$$
M_{j, p}^{3}=\eta_{f g}\left(\Psi^{f}\right)_{j, p}^{*}\left(\left(m^{2}\right)_{h}^{g} \Phi^{h}\right)_{j, p} .
$$

The three extra kinetic terms can be dealt with in the same manner. Their Lorentz invariant expressions are $K_{j}^{\text {supp }}=\sum_{p} K_{j, p}^{\text {supp }}, \Delta K_{j}^{j}=\sum_{p} \Delta K_{j, p}^{j}$, $\Delta K_{j}^{p}=\sum_{p^{\prime}} \Delta K_{j, p^{\prime}}^{p}$, with

$$
\begin{aligned}
K_{j, p}^{\text {supp }}= & \eta_{f g}\left(\Psi^{f}\right)_{j, p}^{*}\left(\left(k^{\text {supp }}\right)_{h}^{g} \nabla_{j} \Phi^{h}\right)_{j, p} \\
\Delta K_{j, p}^{j}= & +\eta_{f g}\left(\Psi^{f}\right)_{j, p}^{*}\left(\left(\Delta k^{j}\right)_{h}^{g} \nabla_{p} \Phi^{h}\right)_{j, p} \\
& \times\left(V_{\mu} \nabla_{j} X^{\mu}\right) \\
\Delta K_{j, p}^{p}= & \eta_{f g}\left(\Psi^{f}\right)_{j, p}^{*}\left(\left(\Delta k_{U}^{p}\right)_{h}^{g} \nabla_{j} \Phi^{h}\right)_{j, p} \\
& \times\left(U_{\mu} \nabla_{p} X^{\mu}\right) \\
- & \eta_{f g}\left(\Psi^{f}\right)_{j, p}^{*}\left(\left(\Delta k_{V}^{p}\right)_{h}^{g} \nabla_{j} \Phi^{h}\right)_{j, p} \\
& \times\left(V_{\mu} \nabla_{p} X^{\mu}\right)
\end{aligned}
$$

where the operators $k^{\text {supp }}, \Delta k^{j}, \Delta k_{U}^{p}, \Delta k_{V}^{p}$ all obey the SOLT law and coincide respectively with $1-T, W \sigma_{3}\left[\left(\sigma_{3}-\bar{\sigma}_{3}\right)+\bar{\sigma}_{3}(T-1)+\left(\sigma_{3}-\bar{\sigma}_{3}\right)(T-1)\right], \sigma_{3}-$ $\bar{\sigma}_{3}$ and $T-1$ in the spin basis of the grid frame. We have also used the standard notations $U_{\mu}=\eta_{\mu \nu} U^{\mu}$ and $V_{\mu}=\eta_{\mu \nu} V^{\mu}$ with $\eta_{\mu \nu}=\operatorname{diag}(1,-1)$, in accordance with the 2-bein components introduced in the previous section. 


\subsection{Stress-energy in an arbitrary Lorentz frame}

Let $\Sigma_{L}$ be the Lorentz invariant action obtained by summing up all previous contributions. By construction, the on-shell values of the functional derivatives of $\Sigma_{L}$ with respect to the derivatives of the coordinates coincide, in the grid reference frame, with the (opposite of) the Hamiltonian $\mathcal{H}$, the momentum $\mathcal{P}$, and their currents $\mathcal{J}_{\mathcal{H}}$ and $\mathcal{J}_{\mathcal{P}}$. These four quantities represent the stress-energy of the DTQW in the grid frame. Because we want to investigate what happens to the stress-energy in other Lorentz frames, we view these quantities as the grid frame components of a single object, the stress-energy 'tensor' $\mathcal{T}$ of the walk. Since the on-shell values of the coordinates in the grid reference frame are $X^{0}=j$ and $X^{1}=p$, we denote the components of the stress energy 'tensor' in the grid frame by $\mathcal{T}_{j}^{j}=\mathcal{H}$, $\mathcal{T}_{j}^{p}=\mathcal{J}_{\mathcal{H}}, \mathcal{T}_{p}^{j}=\mathcal{P}$ and $\mathcal{T}_{p}^{p}=\mathcal{J}_{\mathcal{P}}$. As shown above, these components obey the two conservation equation $\nabla_{j} \mathcal{T}_{j}^{j}+\nabla_{p} \mathcal{T}_{j}^{p}=0$ and $\nabla_{j} \mathcal{T}_{p}^{j}+\nabla_{p} \mathcal{T}_{p}^{p}=0$.

Suppose now we change Lorentz frame to coordinates which we denote by $X^{0}$ and $X^{1}$ for simplicity sake. It is straightforward to show that the onshell values of the derivatives of $\Sigma_{L}$ with respect to the coordinate derivatives coincide on-shell with the Lorentz transforms of the components $\mathcal{T}_{j / p}^{j / p}$ with respect to the lower index (remember that the $C$ 's are the coefficients to be applied on forms when one changes form coordinates $j, p$ to coordinates $\left(X^{0}, X^{1}\right)$, see above):

$$
\begin{aligned}
\mathcal{T}_{0}^{j} & =-\frac{\delta \Sigma}{\delta\left(\nabla_{j} X^{0}\right)} \widehat{=} C_{0}^{j} \mathcal{T}_{j}^{j}+C_{0}^{p} \mathcal{T}_{p}^{j} \\
\mathcal{T}_{0}^{p} & =-\frac{\delta \Sigma}{\delta\left(\nabla_{p} X^{0}\right)} \widehat{=} C_{0}^{j} \mathcal{T}_{j}^{p}+C_{0}^{p} \mathcal{T}_{p}^{p} \\
\mathcal{T}_{1}^{j} & =-\frac{\delta \Sigma}{\delta\left(\nabla_{j} X^{1}\right)} \widehat{=} C_{1}^{j} \mathcal{T}_{j}^{j}+C_{1}^{p} \mathcal{T}_{p}^{j} \\
\mathcal{T}_{1}^{p} & =-\frac{\delta \Sigma}{\delta\left(\nabla_{p} X^{1}\right)} \widehat{=} C_{1}^{j} \mathcal{T}_{j}^{p}+C_{1}^{p} \mathcal{T}_{p}^{p} .
\end{aligned}
$$

These components obey the conservation equations $\nabla_{j} \mathcal{T}_{0}^{j}+\nabla_{p} \mathcal{T}_{0}^{p}=0$ and $\nabla_{j} \mathcal{T}_{1}^{j}+\nabla_{p} \mathcal{T}_{1}^{p}=0$

\section{Continuous limit}

The continuous limit of $2 D$ DTQWs is analyzed in detail in [22]. The most interesting case is the one where the limit exists and coincides with the 
free Dirac equation, which is obtained by collecting all first order terms in the infinitesimal $\epsilon$ which fixes the time- and spatial-step. At first order in $\epsilon$, the operators $\nabla_{j}$ and $\nabla_{p}$ then tend towards $\epsilon \partial_{t}$ and $\epsilon \partial_{x}$, the operator $C$ tends towards unity and the operator $W$ tends towards $1+i \epsilon m \sigma_{1}$ where $\sigma_{1}$ is the first Pauli matrix and $m$ plays the role of the fermion mass. Thus, at first order in $\epsilon, M_{j}^{1}$ coincides with the mass term of the Dirac equation while both $M_{j}^{2}$ and $M_{j}^{3}$ vanish at the same order. As for the kinetic terms,

$\bar{K}_{j}$ coincides at first order in $\epsilon$ with the kinetic term of the Dirac equation while all other kinetic contributions vanish at that order.

\section{Conclusion}

We have shown that the quantum automata linear unitary dynamics derives from a Hamiltonian preserving basic action principle. For DTQWs with constant coefficients on the line, this basic principle can be extended by adding space-time coordinates as new variables defined on the spacetime grid and the equations of motion for these new variables deliver energy and momentum conservation in the grid reference frame in the form of finite differences conservation equations obeyed by the energy and momentum densities together with their flux densities. We have finally proposed a manifestly covariant form of this extended action principle and computed the stress-energy of the DTQW in an arbitrary reference frame. The Lorentz invariance of the 2D Dirac equation has been revisited in Appendix C, while Appendix B shows how charge conservation for DTQWs can be recovered from the action principle. Appendix A presents the basics of discrete action principles on simple examples from classical mechanics. Though Appendix $\mathrm{A}$ is presented as a tutorial, all the material offered in it is new.

Let us now comment on these results and mention a few extensions. The first, very general conclusion of this manuscript is that the dynamics of several physically interesting discrete dynamical systems like quantum automata and in particular DTQWs does derive from action principles. The perhaps unexpected point is that, in the discrete case, the existence of an autonomous i.e. time-independent Lagrangian does not guarantee energy conservation, except in very particular systems like quantum automata. For DTQWs, the Hamiltonian obtained from the basic action principle introduced in this article is essentially the average of the imaginary exponential of the so-called pseudo Hamiltonian already introduced in the literature independently of action principles. The momentum of the DTQW is the sine of the wave-number. 
These two quantities represent physically the energy and the momentum of the DTQW in what we have called the grid reference frame. Associated spatial densities and currents can be also computed by choosing the right variables in the action.

The basic action principle valid for general quantum automata, as specialised to DTQWs, presents two limitations. First, the action involves only the grid coordinates $(j, p)$ and does not involve arbitrary space-time coordinates, so Lorentz invariance cannot even be discussed properly. Second, energy conservation is not generically valid for discrete action principles. Thus, extending the DTQW action to describe for example the dynamics of gauge fields acting on DTQWs would lead to a global dynamics whose Lorentz covariance cannot be discussed and which does not conserve any energy.

As explained in Appendix A, the key to solving both problems lies in introducing space-time coordinates $X^{0}$ and $X^{1}$ as new variables in extended actions. Before discussing this idea, let us review rapidly the previous efforts that have been made $[31,32,33]$ to address Lorentz invariance of DTQWs without introducing new space-time coordinates.

It is obvious that a discrete $2 D$ grid in space-time is not invariant under Lorentz transformations and that is the main problem encountered when trying to discuss Lorentz invariance of any theory defined on a discrete grid. The literature on DTQWs offers two solutions. The first solution [31] is to replace the concept of discrete space-time points by light-like rectangular space-time patches. This however is not sufficient to ensure Lorentz invariance for DTQWs, but it is enough to prove that extensions of DTQWs named Clock DTQWs are exactly Lorentz invariant.

The other solution $[32,33]$ offered by the literature is to switch to Fourier space, which is continuous if the space-time grid is not bounded, and describe Lorentz transformations as non-linear transformations in that space. Thus, in this approach, Lorentz transformations act on components in Fourier space and do not act on coordinates in physical $2 D$ space-time.

The solution offered in the present article in different and much more conventional. In particular, discrete space-time points are not replaced by space-time patches and Lorentz transformations, as usual, act linearly on space-time coordinates. This can be achieved by introducing space-time coordinates are as functions defined on the discrete space-time grid. It turns out that this procedure can also be used to extend the energy- and momentumpreserving basic DTQW action into more general energy- and momentum- 
preserving actions for DTQWs coupled self-consistenly to gauge fields. Let us now discuss this in detail.

As stated in the preceding paragraph, the first step in our approach consists in introducing space-time coordinates as functions defined on the spacetime grid and to treat these as new dynamical variables of an extended action. The equations of motion then have to be solved, not only for the spinor $\Phi$ (and possible other fields like gauge fields if needed), but also for the space-time coordinates $X^{0}$ and $X^{1}$. If the space-time coordinates are introduced properly, conservation equations for an energy and a momentum for the spinor (and possible other fields) then appear as the equations of motion for $X^{0}$ and $X^{1}$. Thus, in a generic case, space-time coordinates cannot be chosen freely and the solution obeying the equations of motion ensures that an energy and momentum for the remaining variables (spinor etc.) is conserved. In the simple case envisaged in this article i.e. free DTQWs, the original dynamics does conserve energy and momentum. Thus, the equations of motion for the spinor and the space-time coordinates are solved identically by the original DTQW dynamics and the choice $X^{0}=j$ and $X^{1}=p$. As it stands, this first extension of the basic action principle does not bring a lot on table when only DTQWs are considered. But this first extended action can be generalized to describe inhomogeneous DTQWs and a coordinate-dependent kinetic term for other fields like gauge fields can also be added. The resulting equations of motion for the fields combined with the space-time coordinates will then enforce energy and momentum conservation. Thus, the first extension of the basic action solves the second problem above.

Note that, at this stage, the coordinate $X^{0}$ and $X^{1}$ are only arbitrary off-shell i.e. when considered as variables on the extended action, and the coordinate-independent on-shell action obtained by replacing $X^{0}$ by $j$ and $X^{1}$ by $p$ is identical to the basic action obeyed by DTQWs. Thus, at this stage, the coordinates $\left(X^{0}, X^{1}\right)$ are not coordinates in an arbitrary Lorentz frame, but space-time coordinates in the Lorentz frame of the grid. They are arbitrary variables of the extended action and become equal to the grid coordinates by virtue of the equations of motion. Thus, this first extension of the basic action principle still describes physics in the grid reference frame only.

Change of reference frame can be performed by considering a second extension of the basic action, which is obtained by rewriting the first extension into a manifestly covariant manner. Let us now discuss some aspects of the quite involved computation. 
Invariance properties of the Dirac equation are notoriously non trivial conceptually. The Lorentz invariance of the 2D flat space-time Dirac equations is reviewed in Appendix $\mathrm{C}$, with special emphasis on geometrical aspects. One of the non-standard aspects of the presentation is that flat-space time is viewed as a special case of the more general curved space-time.

Under a change of Lorentz frame, wave functions of DTQWs and usual Dirac spinors transform the same way. The same goes for spin-operators acting on DTQWs and Dirac spinors, which all obey what we call the SOLT law. Both actions contain the same basic kinetic term, but the discrete action contains extra kinetic and mass terms which vanish at the continuous limit. The extra kinetic and mass terms can be written in a manifestly covariant manner which involves the time-like 2-velocity of the grid in spacetime and its orthogonal vector field or, if one prefers, i.e. the projector unto the space orthogonal to the velocity field. This sort of dependence is standard when one describes in a covariant manner situations which exhibit a special reference frame. Standard examples are relativistic fluid dynamics, including acoustics and diffusive transport in a relativistic fluid, cosmological structure formation and relativistic Hamiltonian formulations. Indeed, scalar equations of state for relativistic fluids [37, 38] link energy density and pressure in the local rest-frame of the fluid and this quantities are contractions of the stress-energy tensor of the fluid with its local velocity field and with the projector orthogonal to this field. Similarly, relativistic acoustics $[39,40]$ studies perturbations around a given flow caracterized notably by its velocity field. Also, the stochastic equations defining physically realistic relativistic stochastic processes [41] can be written in a manifestly covariant manner which involves the velocity of the fluid in which diffusion takes place. In the same vein, cosmological structure formation [42] relies on a perturbation expansion around an isotropic homogeneous solution of Einstein equation, which exhibits a preferred reference frame [43]. This frame is also identified with the so-called co-moving frame where the CMB appears isotropic [44]. Finally, the Hamiltonian formulation of any relativistic theory, from point-particle dynamics [45] to General Relativity [46] is naturally perfectly covariant and is based on a foliation of space-time, which amounts to choosing a reference frame at each point i.e. a velocity field.

Geometrically speaking, the picture that emerges from this article can be summed up in the following manner. For the Dirac equation, the base space-time is a differential manifold, which is a continuous set of points and coordinate systems are used to label these points by real numbers. For the 
DTQWs, the base space-time is the grid and coordinates are used to label the discrete points of this grid by real numbers. Both dynamics derive from an action principles which can be written in a manifestly covariant manner. The Dirac action principle does not involve a particular velocity field $U$ i.e. it does not involve any preferred reference frame. On the contrary, the DTQW action principle, when written in arbitrary Lorentz coordinates, does involve a quantity which can be interpreted as the velocity of the grid in space-time and whose components become $(1,0)$ if one uses coordinates attached to the grid. Figure 1 sums up how the DTQW operates in an arbitrary Lorentz frame.

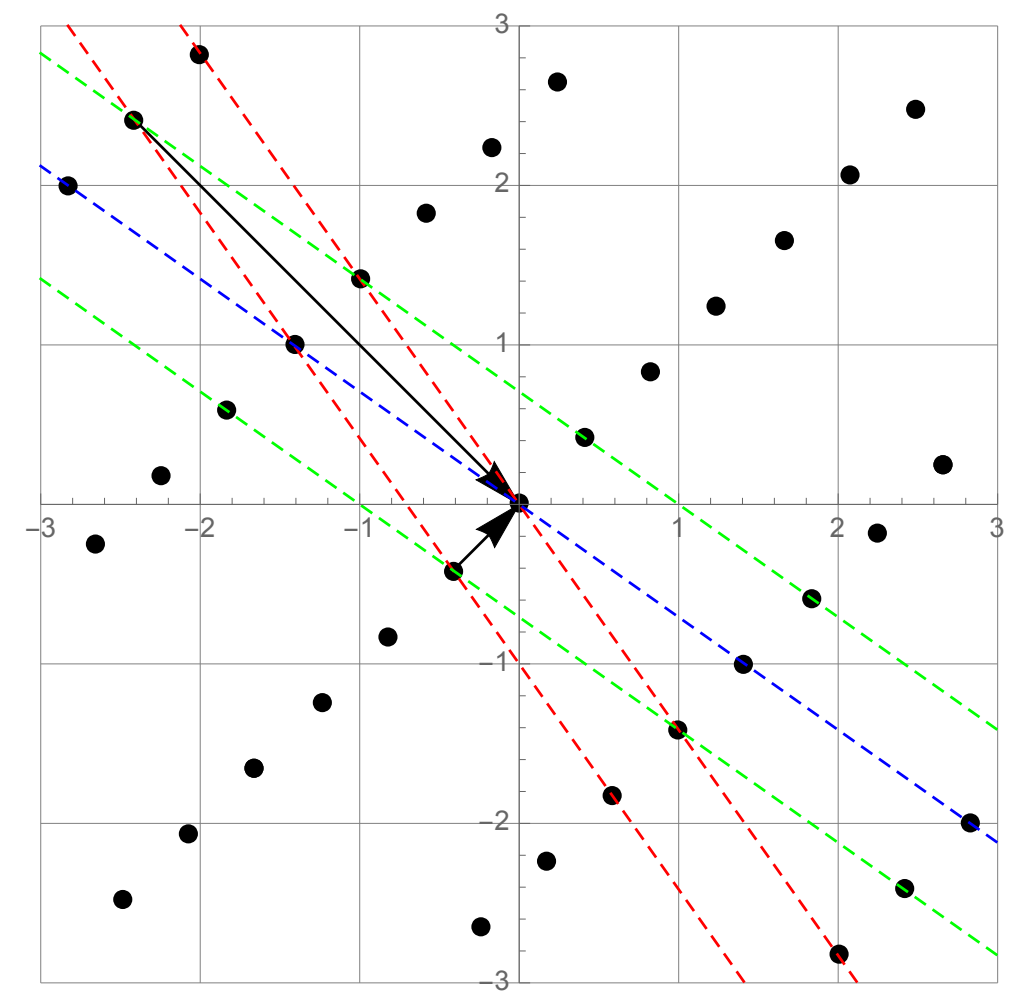

Figure 1: The DTQW in the Lorentz frame with velocity $V=\sqrt{2} / 2$ with respect to the grid frame. The coordinate $X^{0}=\sqrt{2} j-p$ is horizontal and $X^{1}=\sqrt{2} j-p$ is vertical. Grid points corresponding to integer values of $j$ and $p$ are represented by black bullets. The dashed red lines correspond to $j=0$ (right) and $j=-1$ (left). The dashed blue line corresponds to $p=0$ and the dashed green lines correspond to $p=+1$ (right) and $p=-1$ (left). Arrows indicate that the state of the walk at point $j=p=0$ is determined by the state at points $j=-1, p= \pm 1$. 
Let us conclude by mentioning a few natural extensions of this work. The analysis presented in this article should be carried out on more general DTQWs with constant coefficients, not only walks in higher dimensions, but also several step walks. One should then address walks with non constant coefficients, which are essentially discrete models of spinors coupled to gauge fields, and then proceed by adding to the action Lorentz invariant kinetic terms for the gauge fields, thus obtaining energy and momentum conserving discrete models of spinors self-consistently coupled to gauge fields. Finally, action principles can also be used in path-integrals to second quantize DTQWs.

\section{Appendix A. Toolbox for discrete action principles}

\section{Appendix A.1. Basics}

For simplicity, we consider an action $S$ which is a functional of a single real variable $q$ defined on the discrete 'time' line $\mathbb{Z}$. This action can be viewed as as function of the variables $q=\left(q_{j}\right)_{j \in \mathbb{Z}}$. The corresponding equations of motion are thus obtained by enforcing that $S$ is an extremum for arbitrary independent variations of the $q_{j}$ 's. The equations of motion thus read $\forall j \in$ $\mathbb{Z}, \partial S / \partial q_{j}=0$.

Suppose now that the explicit expression of $S$ in terms of the $q_{j}$ makes it natural to write $S$ as a function $\tilde{S}$ of both the $q_{j}$ 's and the finite differences $v_{j}=q_{j+1}-q_{j}=(D q)_{j}$, as is for example the case with the 'mechanical' action

$$
\begin{aligned}
S_{m}(q) & =\sum_{j}\left[\frac{1}{2}\left(q_{j+1}-q_{j}\right)^{2}-\phi\left(q_{j}\right)\right] \\
& =\sum_{j}\left[\frac{1}{2} v_{j}^{2}-\phi\left(q_{j}\right)\right] \\
& =\tilde{S}_{m}(q, v) .
\end{aligned}
$$

The equations of motion can easily be recovered from $\tilde{S}$. Indeed,

$$
\begin{aligned}
\frac{\partial S}{\partial q_{j}} & =\frac{\partial \tilde{S}}{\partial v_{j}} \frac{\partial v_{j}}{\partial q_{j}}+\frac{\partial \tilde{S}}{\partial v_{j-1}} \frac{\partial v_{j-1}}{\partial q_{j}}+\frac{\partial \tilde{S}}{\partial q_{j}} \\
& =-\frac{\partial \tilde{S}}{\partial v_{j}}+\frac{\partial \tilde{S}}{\partial v_{j-1}}+\frac{\partial \tilde{S}}{\partial q_{j}}
\end{aligned}
$$


Introducing the momentum

$$
p_{j}=\frac{\partial \tilde{S}}{\partial v_{j}},
$$

the equation of motion $\partial S / \partial q_{j}=0$ thus reads

$$
(D p)_{j-1}=p_{j}-p_{j-1}=\frac{\partial \tilde{S}}{\partial q_{j}}
$$

which closely parallels the usual Euler-Lagrange equation.

Suppose now that one chooses for example $v_{j}^{\prime}=q_{j+1}-q_{j-1}=\left(D^{\prime} q\right)_{j}$ as velocity and work with an $\tilde{S}^{\prime}\left(v^{\prime}, q\right)$. A similar computation delivers the equation of motion for $q_{j}$ under the form

$$
\left(D^{\prime} p^{\prime}\right)_{j}=p_{j+1}^{\prime}-p_{j-1}^{\prime}=\frac{\partial \tilde{S}^{\prime}}{\partial q_{j}}
$$

with

$$
p_{j}^{\prime}=\frac{\partial \tilde{S}^{\prime}}{\partial v_{j}^{\prime}} .
$$

If one of the $q_{j}$ 's is a cyclic variables of $\tilde{S}$ (resp. $\left.\tilde{S}^{\prime}\right)$ i.e. if $\tilde{S}\left(\right.$ resp. $\left.\tilde{S}^{\prime}\right)$ does not depend on that variable, its conjugate momentum $p_{j}\left(\right.$ resp. $\left.p_{j}^{\prime}\right)$ is conserved in the sense that $(D p)_{j}=0\left(\operatorname{resp} .\left(D^{\prime} p^{\prime}\right)_{j}=0\right)$.

Appendix A.2. Hamiltonian

Consider now the Legendre transform $\tilde{S}_{H}=\sum_{j} p_{j} v_{j}-\tilde{S}$ of $\tilde{S}$. By definition, $\widetilde{S}_{H}$ is a function of the $\left(q_{j}, p_{j}\right)$ 's and its differential reads

$$
d \tilde{S}_{H}=\sum_{j}\left[-\frac{\partial \tilde{S}}{\partial q_{j}} d q_{j}+v_{j} d p_{j}\right] .
$$

Here, $d$ denotes the usual differential and is not connected with any discrete derivative: for each discrete value of $j$, the variables $q_{j}$ and $p_{j}$ are continuous and the function $\tilde{S}_{H}$ can therefore be differentiated with respect to its variables. Combining this with the definition of $v_{j}=q_{j+1}-q_{j}$ and with the equation of motion (A.4) leads to

$$
\begin{aligned}
(D p)_{j-1} & =-\frac{\partial \tilde{S}_{H}}{\partial q_{j}} \\
(D q)_{j} & =\frac{\partial \tilde{S}_{H}}{\partial p_{j}}
\end{aligned}
$$


One can show in a similar way that

$$
\begin{aligned}
\left(D^{\prime} p^{\prime}\right)_{j} & =-\frac{\partial \tilde{S}_{H}^{\prime}}{\partial q_{j}} \\
\left(D^{\prime} q\right)_{j} & =\frac{\partial \tilde{S}_{H}^{\prime}}{\partial p_{j}}
\end{aligned}
$$

where $\tilde{S}_{H}^{\prime}$ is the Legendre transform of $\tilde{S}^{\prime}$ with respect to the $v_{j}^{\prime}$ 's.

Suppose now that the action $S$ does not depend explicitly on $j$ and that $\tilde{S}_{H}(p, q)=\sum_{j} H\left(p_{j}, q_{j}\right)$. The discrete equation (A.8) transcribes into

$$
\begin{aligned}
(D p)_{j-1} & =-\frac{\partial H}{\partial q_{j}} \\
(D q)_{j} & =\frac{\partial H}{\partial p_{j}}
\end{aligned}
$$

which closely ressembles the Hamiltonian or symplectic form of usual continuous equations of motion. But in the discrete case, this fact is not enough to guarantee that the Hamiltonian $H$ is conserved. In the continuous case, conservation of the Hamiltonian can be proven by inserting the symplectic equations of motion into a Taylor-expansion of $H(p+d p, q+d q)$ :

$$
\begin{aligned}
H(p+d p, q+d q) & =H(p, q)+d p \frac{\partial H}{\partial p}+d q \frac{\partial H}{\partial q} \\
& =H(p, q)+\left(-\frac{\partial H}{\partial q} d t\right) \frac{\partial H}{\partial p}+\left(\frac{\partial H}{\partial p} d t\right) \frac{\partial H}{\partial q} \\
& =H(p, q) .
\end{aligned}
$$

In the discrete case, $H\left(p_{j+1}, q_{j+1}\right)=H\left(p_{j}+(D p)_{j}, q_{j}+(D q)_{j}\right)$ but $(D p)_{j}$ and $(D q)_{j}$ are not infinitesimal, so the Taylor expansion makes no sense and conservation of $H$ cannot be proven. Indeed, $H$ is not generally conserved. Take for example the action $S_{m}$, for which $\tilde{S}_{m H}(q, p)=\sum_{j}\left[p_{j}^{2} / 2+\phi\left(q_{j}\right)\right]$ and which generates for all $j$ the equations of motion $(D p)_{j-1}=-\partial \phi / \partial q_{j}$ and $(D q)_{j}=p_{j}$. Then,

$$
H_{j+1}-H_{j}=\phi\left(q_{j}+(D q)_{j}\right)-\phi\left(q_{j}\right)-p_{j}\left(\frac{\partial \phi}{\partial q}\right)_{j+1}+\frac{1}{2}\left(\frac{\partial \phi}{\partial q}\right)_{j+1}^{2}
$$

which only vanishes for constant potentials $\phi$ i.e. for free particles. Thus, $S_{m}$ is a symplectic discretization of the motion of a point particle in the potential 
$\phi$ i.e. the Hamiltonian $H(p, q)$ can be used to write the discrete equations of motion, but this Hamiltonian is not conserved, except in the uninteresting case of constant potential.

\section{Appendix A.3. Conserved energy}

In the discrete case, the Hamiltonian is generally not conserved and therefore does not generally correspond to a conserved energy (though it always is a tool to write down the equations of motion in the so-called symplectic form). As shown in Section ..., a noteworthy exception is the case of DTQWs with constant unitary. These describe physically DTQWs coupled to constant, imposed discrete gauge fields. If one wants to study situations where DTQWs and fields are coupled in a self-consistent manner, one needs to introduce discrete kinetic terms for the fields and these make the total (discrete) Hamiltonian of the DTQWs and the discrete fields not conserved. It is thus both instructive and useful to present a systematic method making it possible to build extensions of the above discrete actions which do conserve an energy when other objects than the DTQWs, for example discrete gauge fields, are allowed their own dynamics and thus, treated as dynamical variables in the action. This can be done by introducing a new 'time' variable $t_{j}$.

Consider first for example an action of the form $\tilde{S}(q, v)=\sum_{j} \tilde{s}\left(q_{j}, v_{j}\right)$, which is autonomous i.e. does not depend explicitly on the discrete time variable $j$, and build from $\tilde{S}$ the following action

$$
\tilde{\Sigma}(q, t, v, V)=\sum_{j} \tilde{s}\left(q_{j}, v_{j} / V_{j}\right) V_{j}=\sum_{j} \tilde{s}\left(q_{j}, u_{j}\right) V_{j},
$$

where $V_{j}=(D t)_{j}$. In passing from $\tilde{S}$ to $\tilde{\Sigma}, \Delta j=(j+1)-j=1$ has been formally replaced by $\Delta t=t_{j+1}-t_{j}=V_{j}$ and the velocity $v_{j}=D q_{j} /(\Delta j)$ has been replaced by $u_{j}=D q_{j} /(\Delta t)=v_{j} / V_{j}$.

The momenta $\pi_{j}$ and $\Pi_{j}$ conjugate to $q_{j}$ and $t_{j}$ read:

$$
\begin{aligned}
\pi_{j} & =\frac{\partial \tilde{\Sigma}}{\partial v_{j}}=V_{j}\left(\frac{\partial \tilde{\sigma}}{\partial u}\right)_{u_{j}=v_{j} / V_{j}} \\
\Pi_{j} & =\frac{\partial \tilde{\Sigma}}{\partial V_{j}}=\tilde{\sigma}\left(u_{j}, q_{j}\right)-u_{j}\left(\frac{\partial \tilde{\sigma}}{\partial u}\right)_{u_{j}=v_{j} / V_{j}}
\end{aligned}
$$

The Legendre transform of $\tilde{\Sigma}$ with respect to the $q$ 's and $t$ 's vanishes identically i.e. there is no non vanishing Hamiltonian to write the equations of 
motion in the form (A.10). On the other hand, the time $t$ is a cyclic variable of the action, so its momentum $\Pi$ is conserved i.e. $\Pi_{j}$ does not depend on $j$. Now, the second equation in (A.14) shows that $\Pi_{j}$ is the Legendre transform of $\tilde{S}$ with respect to the velocity $u_{j}$ and, thus, represents the energy at time $t_{j}$. One thus gets energy conservation as the equation of motion for the time variable $t$ and the actual equation of motion for $q$ is

$$
(D p)_{j-1}=V_{j}\left(\frac{\partial \tilde{\sigma}}{\partial q}\right)_{q_{j}}
$$

Taking again the action $S_{m}$ as an example,

$$
\begin{gathered}
\tilde{\Sigma}_{m}(q, t, v, V)=\sum_{j}\left[\frac{1}{2}\left(\frac{v_{j}}{V_{j}}\right)^{2}-\phi\left(q_{j}\right)\right] V_{j}, \\
\pi_{j}=\frac{v_{j}}{V_{j}}=\frac{(D q)_{j}}{(D t)_{j}} \\
\Pi_{j}=-\frac{1}{2}\left(\frac{(D q)_{j}}{(D t)_{j}}\right)^{2}-\phi\left(q_{j}\right)
\end{gathered}
$$

and the equation of motion for $q_{j}$ reads

$$
\left(D \frac{D q}{D t}\right)_{j-1}=-(D t)_{j}\left(\frac{\partial \phi}{\partial q}\right)_{j} .
$$

Let us comment briefly on the philosophy behind the method we just introduced. The original discrete actions generate an Hamiltonian with which one can write the discrete equations of motion in the so-called symplectic form, but this Hamiltonian is generally not conserved, even for actions which do not depend explicitly on the discrete 'time' $j$, and thus, this Hamiltonian does not correspond to an energy. However, one can build from these non energy conserving actions new discrete actions which involve, not only the original dynamical variables, but a new dynamical variable $t$ identified with the physical time. The equations of motion derived from these new actions cannot be put in symplectic form (because their Hamiltonian identically vanishes), but there is a conserved quantity, the momentum of the time coordinate $t$, which can be identified with the physical energy. In this 
approach, the physical time $t$ does not generally coincide with the grid coordinate $j$. An exception to this is the dynamics of DTQWs coupled to exterior, imposed gauge fields where $t$ can be chosen identical to $j$ (see Sections...). Note however that, even in this case, introducing a new variable $t$ and an accompanying new space variable $x$ defined on the space-time grid makes it possible to change these variables and, in particular, perform Lorentz transformations, even in discrete physics defined on space-time grids. One can then define a Lorentz transformation which acts on the physical coordinates and the spinor wave-function, which are all defined on the fixed space-time grid, which itself remains unchanged under Lorentz transformation.

Suppose now that the function $\tilde{\sigma}$ and, thus, the action $\tilde{\Sigma}$ depend explicitly on $t$ :

$$
\tilde{\Sigma}(q, t, v, V)=\sum_{j} \tilde{\sigma}\left(t_{j}, q_{j}, v_{j} / V_{j}\right) V_{j}=\sum_{j} \tilde{\sigma}\left(t_{j}, q_{j}, u_{j}\right) V_{j}
$$

The equation of motion for $q$ is not modified but the equation of motion for $t$ now reads:

$$
(D \Pi)_{j-1}=\left(\frac{\partial \tilde{\sigma}}{\partial t}\right)_{j} .
$$

The energy is not conserved because the action itself depends on $t$. This kind of action is useful in describing a system (variable $q$ ) evolving under the influence of an external (i.e. imposed) non constant force field. Note that a complete consistent treatment including both $q$ and the field as dynamical variables only involves time (and space-) independent Lagrangian (densities). Note also that, in general, the natural time variable for $\tilde{\sigma}$ is $t$, not the iteration index $j$.

\section{Appendix B. Charge Conservation for DTQWs on the line}

Let us first consider, for simplicity sakes, the so-called one-step $(1+1) \mathrm{D}$ DTQWs with two component wave functions $\Psi=\left(\psi^{-}, \psi^{+}\right)^{\dagger}$, for which $U_{j}=$ $V_{j} T$ where $T$ is the $j$-independent spatial translation operator $T$ defined by

$$
\begin{aligned}
& (T \psi)_{p}^{-}=\psi_{p+1}^{-} \\
& (T \psi)_{p}^{+}=\psi_{p-1}^{+}
\end{aligned}
$$

and $V_{j}$ is defined

$$
\left(V_{j} \Psi\right)_{p}=W_{j, p} \Psi_{p}
$$


with $W_{j, p}$ an arbitrary $j$ - and $p$-dependent operator in $U(2)$.

Let $\left(\rho^{ \pm}, \delta^{ \pm}\right)$be the modulus and phase of the component $\psi^{ \pm}$and introduce the new phases $\mu=\left(\delta^{+}+\delta^{-}\right) / 2$ and $\delta=\left(\delta^{+}-\delta^{-}\right) / 2$. Since $\phi^{ \pm}=(\mu \pm \delta)$, the two-component spinor thus reads

$$
\left(\begin{array}{c}
\psi^{-} \\
\psi^{+}
\end{array}\right)=e^{i \mu}\left(\begin{array}{c}
\rho^{-} e^{-i \delta} \\
\rho^{+} e^{+i \delta}
\end{array}\right)
$$

The equation of motion for each of the $\mu_{j, p}$ 's reads $\partial S / \partial \mu_{j, p}=0$.

We will now show that the action (4) does not involve the variables $\mu_{j, p}$ perse but only linear combinations which are discrete equivalents of their time- and space-derivatives. The contribution $\sum_{j}<\Psi_{j+1}\left|\Psi_{j+1}\right\rangle$ is trivially independent of the $\mu_{j, p}$ 's so let us focus on $\sum_{j}<\Psi_{j+1} \mid U_{j} \Psi_{j}>$. Now,

$$
\begin{aligned}
\left(T \Psi_{j}\right)_{j, p} & =\left(\begin{array}{c}
\psi_{j, p+1}^{-} \\
\psi_{j, p-1}^{+}
\end{array}\right) \\
& =e^{i \tilde{\mu}_{j, p}}\left(\begin{array}{c}
\rho_{j, p+1}^{-} e^{-i \tilde{\delta}_{j, p}} \\
\rho_{j, p-1}^{+} e^{+i \tilde{\delta}_{j, p}}
\end{array}\right)
\end{aligned}
$$

where

$$
\tilde{\mu}_{j, p}=\frac{\mu_{j, p+1}+\mu_{j, p-1}-\delta_{j, p+1}+\delta_{j, p-1}}{2}
$$

and

$$
\tilde{\delta}_{j, p}=\frac{-\mu_{j, p+1}+\mu_{j, p-1}+\delta_{j, p+1}+\delta_{j, p-1}}{2} .
$$

It follows that the action (4) only depends on the $\delta_{j, p}$ 's and on

$$
\left(\nabla_{p} \mu\right)_{j, p}=\frac{\mu_{j, p+1}-\mu_{j, p-1}}{2}
$$

and

$$
\left(\nabla_{j} \mu\right)_{j, p}=\mu_{j+1, p}-\frac{\mu_{j, p+1}+\mu_{j, p-1}}{2} .
$$

The variables $\mu_{j, p}$ are thus cyclic. There is therefore a corresponding conserved charge and the associated conserved current $\left(J_{j}, J_{p}\right)$ can be computed from the action (4) by the same method as the one described in the Appendix on the simpler case where all quantities depend only on the discrete time $j$. Indeed, the above equation of motion for $\mu_{j, p}$ can be written as

$$
\left(\nabla_{j}^{\prime} J_{j}\right)_{j, p}+\left(\nabla_{p} J_{p}\right)_{j, p}=0
$$


where

$$
\begin{gathered}
\left(\nabla_{p} J_{p}\right)_{j, p}=\left(J_{p}\right)_{j, p+1}-\left(J_{p}\right)_{j, p-1} \\
\left(\nabla_{j}^{\prime} J_{j}\right)_{j, p}=\frac{\left(J_{j}\right)_{j, p+1}+\left(J_{j}\right)_{j, p-1}}{2}-\left(J_{j}\right)_{j-1, p}
\end{gathered}
$$

and

$$
\begin{aligned}
\left(J_{p}\right)_{j, p} & =\frac{\partial S}{\partial\left(\nabla_{p} \mu\right)_{j, p}}, \\
\left(J_{j}\right)_{j, p} & =\frac{\partial S}{\partial\left(\nabla_{j} \mu\right)_{j, p}} .
\end{aligned}
$$

In the last two equations, $S$ stands for the action (4) understood as a function of the $\left(\nabla_{j} \mu\right)_{j, p}$ 's and $\left(\nabla_{p} \mu\right)_{j, p}$ 's, and not of the $\mu_{j, p}$ 's.

A direct computation leads to

$$
\begin{aligned}
& \left(J_{j}\right)_{j, p}=+\left(\rho_{j, p}^{-}\right)^{2}+\left(\rho_{j, p}^{+}\right)^{2} \\
& \left(J_{p}\right)_{j, p}=-\left(\rho_{j, p}^{-}\right)^{2}+\left(\rho_{j, p}^{+}\right)^{2}
\end{aligned}
$$

which are the usual expressions for the spatial density and current of the DTQW [26].

\section{Appendix C. Lorentz invariance of the $2 D$ flat space-time Dirac action}

In an arbitrary given Lorentz frame $\mathcal{F}$, the flat space-time $2 D$ Dirac Lagrangian density reads [47]

$$
L\left[\Psi, \Psi^{\dagger}\right]=\eta_{\sigma \delta}\left(\Psi^{\delta}\right)^{*}\left(e_{a}^{\mu} \gamma^{0} \gamma^{a} \partial_{\mu}+i m \gamma^{0}\right)_{\omega}^{\sigma} \Psi^{\omega}
$$

where the $\Psi^{\sigma}$ 's are the component of $\Psi$ on the spin space basis $\left(b_{-}, b_{+}\right)$of $\mathcal{F}$ and the components of the metric $\eta$ in spin space are $\eta_{\sigma \delta}=1$ if $\sigma=\delta$ and 0 otherwise. The $e_{a}^{\mu}$ 's are the 2-bein coefficients which we choose to be $e_{0}^{t}=e_{1}^{x}=1, e_{1}^{t}=e_{0}^{x}=0$ and we retain a representation where

$$
\begin{aligned}
& \left(\gamma^{0}\right)_{\delta}^{\sigma}=\left(\begin{array}{ll}
0 & 1 \\
1 & 0
\end{array}\right) \\
& \left(\gamma^{1}\right)_{\delta}^{\sigma}=\left(\begin{array}{cc}
0 & 1 \\
-1 & 0
\end{array}\right),
\end{aligned}
$$


so that $\left(\gamma^{0}\right)^{2}=-\left(\gamma^{1}\right)^{2}=1, \gamma^{0} \gamma^{1}=\operatorname{diag}(-1,1)=-\sigma_{3}$ and $\left(\eta \cdot \gamma_{0}\right)_{\delta \omega}=$ $\eta_{\sigma \delta}\left(\gamma^{0}\right)_{\omega}^{\sigma}=1$ if $\delta \neq \omega$ and 0 otherwise. Making all this explicit in the Dirac Lagrangian delivers:

$$
\begin{aligned}
L\left[\Psi, \Psi^{\dagger}\right]= & \left(\Psi^{-}\right)^{*}\left(+\partial_{t}-\partial_{x}\right) \Psi^{-} \\
& +\left(\Psi^{+}\right)^{*}\left(+\partial_{t}+\partial_{x}\right) \Psi^{+} \\
& +i m\left[\left(\Psi^{-}\right)^{*} \Psi^{+}+\left(\Psi^{+}\right)^{*} \Psi^{-}\right] .
\end{aligned}
$$

Writing the Dirac equation in the so-called Schrödinger form $\partial_{t} \Psi=U_{D} \Psi$ with $\left(U_{D} \Psi\right)^{-}=\partial_{x} \Psi^{-}-i m \Psi^{+}$and $\left(U_{D} \Psi\right)^{+}=-\partial_{x} \Psi^{+}-i m \Psi^{-}$makes it possible to put the Dirac Lagrangian under the form

$$
\begin{aligned}
L\left[\Psi, \Psi^{\dagger}\right]= & \left(\Psi^{-}\right)^{*}\left(\partial_{t} \Psi^{-}-\left(U_{D} \Psi\right)^{-}\right) \\
& +\left(\Psi^{+}\right)^{*}\left(\partial_{t} \Psi^{+}-\left(U_{D} \Psi\right)^{+}\right) .
\end{aligned}
$$

This form is closely parallels the action (4) from which the quantum automata dynamics derives.

Suppose now one performs a change of basis in spin-space and define the new basis vectors $b_{\sigma^{\prime}}$ by $b_{-^{\prime}}=\lambda b_{-}, b_{+^{\prime}}=b_{+} / \lambda$, where $\lambda$ is a non-vanishing real constant. The components of $\Psi$ in this new basis are $\Psi^{-^{\prime}}=\Psi^{-} / \lambda$ and $\Psi^{+^{\prime}}=\lambda \Psi^{+}$and the operators $\gamma^{0}$ and $\gamma^{1}$ are represented by the matrices

$$
\begin{aligned}
\left(\gamma^{0}\right)_{\delta^{\prime}}^{\sigma^{\prime}} & =\left(\begin{array}{cc}
0 & \lambda^{-2} \\
\lambda^{2} & 0
\end{array}\right) \\
\left(\gamma^{1}\right)_{\delta^{\prime}}^{\sigma^{\prime}} & =\left(\begin{array}{cc}
0 & \lambda^{-2} \\
-\lambda^{2} & 0
\end{array}\right) .
\end{aligned}
$$

Similarly, $\eta_{-^{\prime}-^{\prime}}=\lambda^{2}, \eta_{+^{\prime}+^{\prime}}=\lambda^{-2}$ and the other components vanish. This leaves the matrix representations of $\left(\gamma^{0}\right)^{2}=1,\left(\gamma^{1}\right)^{2}=-1, \gamma^{0} \gamma^{1}$ and $\eta \cdot \gamma^{0}$ unchanged.

The basis change thus preserves the Clifford algebra and therefore amounts to a change of representation. One obtains, either from (C.3) or directly from (C.1) leaving the 2-bein unchanged:

$$
\begin{aligned}
L\left[\Psi, \Psi^{\dagger}\right]= & \lambda^{2}\left(\Psi^{-^{\prime}}\right)^{*}\left(+\partial_{t}-\partial_{x}\right) \Psi^{{{ }^{\prime}}^{\prime}} \\
& +\frac{1}{\lambda^{2}}\left(\Psi^{+^{\prime}}\right)^{*}\left(+\partial_{t}+\partial_{x}\right) \Psi^{+^{\prime}} \\
& +i m\left[\left(\Psi^{-^{\prime}}\right)^{*} \Psi^{+^{\prime}}+\left(\Psi^{+^{\prime}}\right)^{*} \Psi^{-^{\prime}}\right] .
\end{aligned}
$$


This is identical to (C.3), except for the $\lambda^{ \pm 2}$ factors. These factors come from the components of the spin metric $\eta$ in the new basis (remember that the matrix representing $\left(\gamma^{0}\right)^{2}=1$ and $\gamma^{0} \gamma^{1}$ are unchanged by the change of basis). But, looking only at (C.6) and (C.1) together, one could be tempted to interpret these two $\lambda^{ \pm 2}$ factors as coming from the 2-bein, and not the components of the metric $\eta$.

Indeed, consider the new spin-metric $\eta^{\prime}$ defined by its components $\eta_{\sigma^{\prime} \delta^{\prime}}^{\prime}=$ 1 if $\sigma^{\prime}=\delta^{\prime}$ and 0 otherwise, the new operators $\left(\gamma^{\prime}\right)^{0}$ and $\left(\gamma^{\prime}\right)^{1}$ defined by

$$
\begin{aligned}
& \left(\left(\gamma^{\prime}\right)^{0}\right)_{\delta^{\prime}}^{\sigma^{\prime}}=\left(\begin{array}{ll}
0 & 1 \\
1 & 0
\end{array}\right) \\
& \left(\left(\gamma^{\prime}\right)^{1}\right)_{\delta^{\prime}}^{\sigma^{\prime}}=\left(\begin{array}{cc}
0 & 1 \\
-1 & 0
\end{array}\right),
\end{aligned}
$$

and the new 2-bein defined by its components on the coordinate basis $\left(\partial_{t}, \partial_{x}\right)$ :

$$
\begin{aligned}
& \left(e^{\prime}\right)_{0}^{t}=\left(+\lambda^{2}+\lambda^{-2}\right) / 2 \\
& \left(e^{\prime}\right)_{0}^{x}=\left(-\lambda^{2}+\lambda^{-2}\right) / 2 \\
& \left(e^{\prime}\right)_{1}^{t}=\left(-\lambda^{2}+\lambda^{-2}\right) / 2 \\
& \left(e^{\prime}\right)_{1}^{x}=\left(+\lambda^{2}+\lambda^{-2}\right) / 2
\end{aligned}
$$

The triplet $\left(\eta^{\prime}, \gamma^{\prime}, e^{\prime}\right)$ delivers the same Lagrangian (C.6) as the triplet $(\eta, \gamma, e)$.

Now, we have not changed space-time coordinates so far and the new 2bein $e^{\prime}$ has been introduced by its components on the original tangent basis $\partial_{\mu}$. As well known, it is possible to introduce new coordinates $x^{\mu^{\prime}}$ and the associated coordinate basis $\partial_{x^{\mu^{\prime}}}$ where the non vanishing of the components of the 2-bein $e^{\prime}$ are $\left(e^{\prime}\right)_{0}^{t^{\prime}}=\left(e^{\prime}\right)_{1}^{x^{\prime}}=1$. Using these coordinates, the Dirac Lagrangian reads

$$
\begin{aligned}
L\left[\Psi, \Psi^{\dagger}\right] & =\left(\Psi^{-^{\prime}}\right)^{*}\left[\left(+\partial_{t^{\prime}}-\partial_{x^{\prime}}\right) \Psi^{-^{\prime}}+i m \Psi^{+^{\prime}}\right] \\
& +\left(\Psi^{+^{\prime}}\right)^{*}\left[\left(+\partial_{t^{\prime}}+\partial_{x^{\prime}}\right) \Psi^{+^{\prime}}+i m \Psi^{-^{\prime}}\right],
\end{aligned}
$$

which is formally identical to (C.3).

Note that, at fixed mass $m$, the change of $\gamma$ operators performed above may be considered as collateral to, or induced by the change of spin metric. 
Indeed, at fixed value of $m$, keeping the mass term fixed comes down to keeping $\eta \cdot \gamma$ constant and thus, changing $\eta$ makes it necessary to change the $\gamma$ operators. Note also that the 2-bein defines the space-time metric, so a change of 2-bein is actually a change of space-time metric. A Lorentz transformation is thus basically a change of spin basis compensated by or compensating a change of coordinate basis/coordinate system through a combined change of spin metric (together with the induced change of $\gamma$ operators) and space-time metric.

All this can be seen in a perhaps more compact way. Consider expressions of the form

$$
F\left[\Psi, \Psi^{\dagger}\right]=\eta_{\sigma \delta}\left(\Psi^{\delta}\right)^{*} O_{\omega}^{\sigma} \Psi^{\omega} .
$$

The Lagrangian (C.1) corresponds to $O_{D}=e_{a}^{\mu} \gamma^{0} \gamma^{a} \partial_{\mu}+i m \gamma^{0}$. Consider the same two spin bases and the same two spin metrics as above. It is simple to check that $F$ can be rewritten as

$$
F\left[\Psi, \Psi^{\dagger}\right]=\left(\eta^{\prime}\right)_{\sigma^{\prime} \delta^{\prime}}\left(\Psi^{\delta^{\prime}}\right)^{*}\left(O^{\prime}\right)_{\omega^{\prime}}^{\sigma^{\prime}} \Psi^{\omega^{\prime}}
$$

with

$$
\begin{aligned}
O_{L^{\prime}}^{L^{\prime}} & =\lambda^{+2} O_{L}^{L} \\
O_{R^{\prime}}^{R^{\prime}} & =\lambda^{-2} O_{R}^{R} \\
O_{R^{\prime}}^{L^{\prime}} & =O_{R}^{L} \\
O_{L^{\prime}}^{R^{\prime}} & =O_{L}^{R} .
\end{aligned}
$$

We call this the Spin Operator Lorentz Transformation (SOLT) law. Now apply the SOLT law to the mass term in $O_{D}$. The operator $\gamma^{0}$ is represented by an anti-diagonal matrix so, at constant $m$, the components of $\left(\gamma^{\prime}\right)^{0}$ in the prime basis must be the same as those of $\gamma^{0}$ in the original basis. The Clifford algebra then fixes $\left(\gamma^{\prime}\right)^{1}$. One remarks that the prime components of $\left(\gamma^{\prime}\right)^{a}$ are identical to the original components of $\gamma^{a}$.

If one now turns to the kinetic term in $O_{D}$, both operators $\left(e_{0}^{\mu} \partial_{\mu}\right)\left(\gamma^{0} \gamma^{0}\right)$ and $\left(e_{1}^{\mu} \partial_{\mu}\right)\left(\gamma^{0} \gamma^{1}\right)$ are represented by diagonal matrices in the original basis. The SOLT law thus generates extra $\lambda^{ \pm 2}$ factors, which do not come from the $\left(\gamma^{\prime}\right)^{a}$. These extra factors must therefore come from the two factors (scalars in spin space) $\left(e_{a}^{\mu} \partial_{\mu}\right)$. This determines the change of 2-bein and, in turn, the change of coordinates (to keep the coordinate components of the 2-bein unchanged). 
The SOLT law is used extensively in the core of this article, where the Lorentz invariance of the action for DTQWs is discussed.

We finally recall that

$$
\left(e^{\prime}\right)_{a}^{\mu^{\prime}}=\frac{\partial x^{\mu^{\prime}}}{\partial x^{\nu}}\left(e^{\prime}\right)_{a}^{\nu}=N_{\nu}^{\mu^{\prime}}\left(e^{\prime}\right)_{a}^{\nu}
$$

so that

$$
\left(e^{\prime}\right)_{a}^{\mu}=\left(N^{-1}\right)_{\nu^{\prime}}^{\mu}\left(e^{\prime}\right)_{a}^{\nu^{\prime}},
$$

where the components of $N^{-1}$ are given by

$$
\begin{aligned}
\left(N^{-1}\right)_{t^{\prime}}^{t} & =+\frac{1}{\delta} \frac{\partial x^{\prime}}{\partial x} \\
\left(N^{-1}\right)_{x^{\prime}}^{t} & =-\frac{1}{\delta} \frac{\partial t^{\prime}}{\partial x} \\
\left(N^{-1}\right)_{t^{\prime}}^{x} & =-\frac{1}{\delta} \frac{\partial x^{\prime}}{\partial t} \\
\left(N^{-1}\right)_{x^{\prime}}^{x} & =+\frac{1}{\delta} \frac{\partial t^{\prime}}{\partial t}
\end{aligned}
$$

with

$$
\delta=\frac{\partial t^{\prime}}{\partial t} \frac{\partial x^{\prime}}{\partial x}-\frac{\partial t^{\prime}}{\partial x} \frac{\partial x^{\prime}}{\partial t} .
$$

At fixed values of the components $\left(e^{\prime}\right)_{a}^{\nu^{\prime}}$, the values of the components $\left(e^{\prime}\right)_{a}^{\mu}$ can thus be encoded in the functions $t^{\prime}(t, x)$ and $x^{\prime}(t, x)$. This is used in the main part of this article to introduce coordinates in the DTQW action.

[1] R.P. Feynman and A.R. Hibbs, Quantum mechanics and path integrals, International Series in Pure and Applied Physics, McGraw-Hill Book Company, 1965.

[2] Y. Aharonov, L. Davidovich and N. Zagury, Phys. Rev. A 48, 1687, 1993.

[3] D.A. Meyers. J. Stat. Phys. 85, 551-574, 1996.

[4] H. Schmitz, R. Matjeschk, Ch. Schneider, J. Glueckert, M. Enderlein, T. Huber and T. Schaetz, Phys. Rev. Lett. 103, 090504, 2009.

[5] F. Zähringer, G. Kirchmair, R. Gerritsma, E. Solano, R. Blatt and C.F. Roos, Phys. Rev. Lett. 104, 100503, 2010. 
[6] A. Schreiber, K.N. Cassemiro, V. Potoček, A. Gábris, P. J. Mosley, E. Andersson, I. Jex and Ch. Silberhorn, Phys. Rev. Lett. 104, 050502, 2010.

[7] M. Karski, L. Förster, J.-M. Cho, A. Steffen, W. Alt, D. Meschede and A. Widera, Science 325(5937), 174- 177, 2009.

[8] L. Sansoni, F. Sciarrino, G. Vallone, P. Mataloni, A. Crespi, R. Ramponi and R. Osellame, Phys. Rev. Lett. 108, 010502, 2012.

[9] B.C. Sanders, S.D. Bartlett, B. Tregenna and P.L. Knight, Phys. Rev. A 67, 042305, 2003.

[10] H.B. Perets, Y. Lahini, F. Pozzi, M. Sorel, R. Morandotti and Y. Silberberg, Phys. Rev. Lett. 100, 170506, 2008.

[11] A. Ambainis, SIAM J. Comput. 37, 210-239, 2007.

[12] F. Magniez, A. Nayak, J. Roland and M. Santha, Search via quantum walk, SIAM J. Comput. - Proceedings of the thirty-ninth annual ACM symposium on Theory of computing, New York, 2007.

[13] C. Aslangul, J. Phys. A 38, 1-16, 2005.

[14] S. Bose, Phys. Rev. Lett. 91, 207901, 2003.

[15] D. Burgarth, University College London, PhD thesis, 2006.

[16] S. Bose, Contemp. Phys. 48, 13-30, 2007.

[17] G. Di Molfetta, F. Debbasch and M. Brachet, Phys. Rev. E 92, 042923, 2015.

[18] E. Collini, C.Y. Wong, K.E. Wilk, P.M.G. Curmi, P. Brumer and G.D. Scholes, Nature 463(7281), 644-647, 2010.

[19] G.S. Engel, T.R. Calhoun, R.L. Read, T.-K. Ahn, T. Manal, Y.C. Cheng, R.E. Blankenship and G.R. Fleming, Nature 446, 782-786, 2007.

[20] C. Cedzich, T. Rybár, A.H. Werner, A. Alberti, M. Genske and R.F. Werner. Phys. Rev. Lett. 111, 160601,2013. 
[21] G. Di Molfetta, M. Brachet and F. Debbasch, Phys. Rev. A 88(4), 042301, 2013.

[22] G. Di Molfetta, M. Brachet and F. Debbasch, Physica A 397, 157-168, 2014.

[23] P. Arrighi, S. Facchini and M. Forets, Quantum Information Processing, 1-20, 2015.

[24] P. Arnault and F. Debbasch, Physica A 443, 179-191, 2016.

[25] L.A. Bru, M. Hinarejos, F. Silva, G.J. de Valcárcel and E. Roldán. Phys. Rev. A 93, 032333, 2016.

[26] P. Arnault and F. Debbasch, Phys. Rev. A 93, 052301, 2016.

[27] P. Arrighi and Ch. Patricot, Quantum Information and Computation 17(9), 2016.

[28] P. Arnault and F. Debbasch, Ann. Phys. 383, 645-661, 2017.

[29] M. Genske, W. Alt, A. Steffen, A.H. Werner, R.F. Werner, D. Meschede and A. Alberti. Phys. Rev. Lett. 110, 190601, 2013.

[30] L. Quentin, Master's Thesis, Sorbonne University, unpublished, 2016.

[31] P. Arrighi, S. Facchini and M. Forets, New J. Phys. 16, 093007, 2014.

[32] A. Bisio, G.M. D'Ariano and P. Perinotti, Phys. Rev. A 94, 042120, 2016.

[33] A. Bisio, G.M. D'Ariano and P. Perinotti, Found. Phys. 47, 1065, 2017.

[34] L. Landau and E. Lifshitz. Relativistic Quantum Theory, Pergamon Press, 1974.

[35] M.J. Ablowitz and A.S. Fokas. Complex Variables, Cambridge University Press, 2003.

[36] P. Arnault, G. Di Molfetta, M. Brachet and F. Debbasch, Phys. Rev. A 94, 012335, 2016. 
[37] S.R. de Groot, W.A. van Leeuwen and C.G. van Weert. Relativistic Kinetic Theory. North-Holland, Amsterdam, 1980.

[38] W. Israel. Covariant fluid mechanics and thermodynamics: An introduction. In A. Anile and Y. Choquet-Bruhat, editors, Relativistic Fluid Dynamics, volume 1385 of Lecture Notes in Mathematics, Springer-Verlag, Berlin, 1987.

[39] L.D. Landau and E.M. Lifshitz. Fluid Mechanics. Elsevier, 2004.

[40] F. Debbasch and M.E. Brachet. Physica D 108, 135, 1997.

[41] C. Chevalier and F. Debbasch, J. Math. Phys. 49(4),043303, 2008.

[42] P.J.E.. Peebles. Principles of Physical Cosmology. Princeton Series in Physics, Princeton University Press, 1993.

[43] S.W. Hawking and G.F.R. Ellis. The large scale structure of space-time. Cambridge Monographs on Mathematical Physics, Cambridge University Press, Cambridge, 1973.

[44] E.W. Kolb and M.S. Turner. The early universe. Frontiers in 69, Westview Press, 1994.

[45] A.O. Barut. Electrodynamics and classical theory of fields and particles. Dover Publications Inc., New York, 1980.

[46] R.M. Wald. General Relativity. The University of Chicago Press, Chicago, 1984.

[47] A. Sinha and R. Roychoudhury. Int. J. Th.Phys. 33(7):1511-1522, 1994. 